\title{
On least-order flow representations for aerodynamics and aeroacoustics
}

\author{
Michael Schlegel ${ }^{1} \dagger$, Bernd R. Noack ${ }^{2}$, Peter Jordan ${ }^{2}$, Andreas Dillmann ${ }^{3}$, \\ Elmar Gröschel ${ }^{4,5}$, Wolfgang Schröder ${ }^{4}$, Mingjun Wei ${ }^{6}$, Jonathan B. Freund ${ }^{7}$, \\ Oliver Lehmann ${ }^{8}$ and Gilead Tadmor ${ }^{8}$ \\ ${ }^{1}$ Institut für Strömungsmechanik und Technische Akustik, Technische Universität Berlin MB1, \\ Straße des 17. Juni 135, D-10623 Berlin, Germany \\ 2 Institut $\mathrm{P}^{\prime}$, CNRS-Université de Poitiers-ENSMA, UPR 3346, Département Fluides, Thermique, \\ Combustion, CEAT, 43 rue de l'Aérodrome, F-86036 Poitiers CEDEX, France \\ ${ }^{3}$ Institut für Aerodynamik und Strömungstechnik, Deutsches Zentrum für Luft- und Raumfahrt, \\ Bunsenstraße 10, D-37073 Göttingen, Germany \\ ${ }^{4}$ Aerodynamisches Institut, Rheinisch-Westfälische Technische Hochschule Aachen, Wüllnerstraße 5a, \\ D-52062 Aachen, Germany \\ 5 ABB Turbo Systems AG, Bruggerstraße 71a, 5400 Baden, Switzerland \\ ${ }^{6}$ Mechanical and Aerospace Engineering, New Mexico State University, PO Box 30001/Dept 3450, \\ Las Cruces, NM 88003-8001, USA \\ ${ }^{7}$ Mechanical Science \& Engineering, University of Illinois at Urbana-Champaign, \\ 1206 West Green Street, Urbana, IL 61801, USA \\ 8 Northeastern University, Department of Electrical and Computer Engineering, \\ 440 Dana Research Building, Boston, MA 02115, USA
}

(Received 26 August 2009; revised 31 October 2011; accepted 3 February 2012;

first published online 16 March 2012)

We propose a generalization of proper orthogonal decomposition (POD) for optimal flow resolution of linearly related observables. This Galerkin expansion, termed 'observable inferred decomposition' (OID), addresses a need in aerodynamic and aeroacoustic applications by identifying the modes contributing most to these observables. Thus, OID constitutes a building block for physical understanding, leastbiased conditional sampling, state estimation and control design. From a continuum of OID versions, two variants are tailored for purposes of observer and control design, respectively. Firstly, the most probable flow state consistent with the observable is constructed by a 'least-residual' variant. This version constitutes a simple, easily generalizable reconstruction of the most probable hydrodynamic state to preprocess efficient observer design. Secondly, the 'least-energetic' variant identifies modes with the largest gain for the observable. This version is a building block for Lyapunov control design. The efficient dimension reduction of OID as compared to POD is demonstrated for several shear flows. In particular, three aerodynamic and aeroacoustic goal functionals are studied: (i) lift and drag fluctuation of a two-dimensional cylinder wake flow; (ii) aeroacoustic density fluctuations measured by a sensor array and emitted from a two-dimensional compressible mixing layer; 
and (iii) aeroacoustic pressure monitored by a sensor array and emitted from a three-dimensional compressible jet. The most 'drag-related', 'lift-related' and 'loud' structures are distilled and interpreted in terms of known physical processes.

Key words: aeroacoustics, low-dimensional models, wakes/jets

\section{Introduction}

The goal of our modelling efforts is to distil a physical understanding of the flow physics enabling flow control of aerodynamic and aeroacoustic observables.

Reduced-order representations of the coherent flow dynamics constitute key enablers of this purpose. The optimum is, of course, represented by analytical formulae for the flow field. Yet, there exist only a small number of corresponding examples, mostly restricted to quasi-steady base flows and periodic flows (Townsend 1956). A more generally applicable strategy for the purposes of flow control is achieved by a low-dimensional flow parametrization. Here, vortex models constitute one of the oldest forms of reduced-order representations. These are well linked to a physical understanding of the flow dynamics and the generation of sound (see e.g. Lugt 1996; Howe 2003; Wu, Ma \& Zhou 2006) considering interacting eddies as the basic flow elements ('particle picture'). However, most control design methods are inhibited by the hybrid nature of vortex models (Pastoor et al. 2008), e.g. the modelling of periodic vortex shedding using a continuous insertion of new state variables representing the locations of the shed vortices. A second form of reduced-order representation is given by Galerkin models, including the Galerkin expansion and the dynamical system for the modal amplitudes. In the Galerkin expansion, the basic flow elements are considered to be spatial structures with time-varying amplitudes ('wave picture'), thus completing a particle-wave analogy of both vortex models and Galerkin models. In comparison to the vortex models, the Galerkin models exhibit a smaller dynamical bandwidth, such that unresolved effects have to be implemented separately using, for example, mean-field, pressure and turbulence models (see e.g. Rempfer \& Fasel 1994; Cazemier, Verstappen \& Veldman 1998; Noack et al. 2003; Noack, Papas \& Monkewitz 2005; Willcox \& Megretski 2005; Noack et al. 2008). However, the simple nature of the Galerkin system of ordinary differential equations enables the straightforward application of a rich kaleidoscope of the methodologies of nonlinear dynamics and control theory. In this paper, the path of Galerkin expansion is pursued for reduced-order representation.

Galerkin expansion modes are derived from various design principles (Noack, Morzyński \& Tadmor 2011). The mathematical property of completeness is guaranteed by 'mathematical modes', which are utilized, for example, in spectral methods for numerical flow computation. A low-order description of the linear flow dynamics is provided by the eigenmodes of linear stability analysis. The eigenmodes of the observability and of the controllability Gramians are most aligned with an observable for given linear dynamics and with control effects, respectively. Finally, modes of the proper orthogonal decomposition (POD) are most fitted to empirical data compression. Here, we follow the empirical approach employing generalizations of POD.

Generalizations of POD have been developed for several purposes. Major emphasis has been laid on data compression of multiple operating conditions such as, for example, sequential POD (Jørgensen, Sørensen \& Brøns 2003), mode interpolation 
(Morzyński et al. 2007) and double POD (Siegel et al. 2008), or the consideration of incomplete data sets (see e.g. Willcox 2006). The focus in this paper is on the manipulation of the utilized POD inner product or norm in the spirit of Freund \& Colonius $(2002$, 2009). But, in our approach, the construction of the employed hydrodynamic function subspace is tailored for purposes of observer and control design.

Examples of decomposition techniques are summarized in table 1. Here, one example is proposed by the balanced POD (BPOD), enabling the numerical approximation of the balanced truncation for linear systems. Here, the inner product or norm of the $\boldsymbol{L}^{2}$ Hilbert space is modified based on the empirical observability Gramian (see e.g. Willcox \& Peraire 2002; Rowley 2005). Moreover, the computation of eigenvectors of the observability Gramian is enabled by the concept of the empirical observability Gramian. Thus observable modes, structures with quantified observability given by the corresponding eigenvalue, are represented. A generalized balanced truncation of nonlinear systems has been proposed by Lall, Marsden \& Glavaški $(1999,2002)$ using generalized empirical Gramians. The generalization of empirical observability Gramians enables the definition of the observable modes to be the eigenfunction of a generalized empirical observability Gramian. However, in aerodynamic and aeroacoustic systems, the identification of observable structures is mostly inhibited by an extensive computational burden needed to provide an ensemble of transients given from a large number of initial conditions.

The starting point of this paper is solely aerodynamic and aeroacoustic databases of the hydrodynamic attractor and the observable describing the kinematics. The definition of observable structures has to be reconsidered, because the observable modes are defined only for asymptotically stable dynamics or for dynamics that can be stabilized under a certain control. This is in general not the case for uncontrolled attractor dynamics. We interpret the extended POD approach (EPOD) as an example for such a redefinition based on the modification of the POD inner product. In EPOD, structures of the hydrodynamic field are identified that are most correlated with a given observable, e.g. with pressure signals beyond the considered domain (Picard \& Delville 2000; Maurel, Borée \& Lumley 2001; Borée 2003; Hoarau et al. 2006). Flow estimation is therefore facilitated by EPOD to reconstruct the hydrodynamic attractor from a measured observable.

In the present paper, a unifying framework termed 'observable inferred decomposition' (OID) of POD generalizations is proposed, modifying the POD inner product or norm and identifying 'OID structures' as kinematic counterparts of most observable structures, the eigenstructures from the observability Gramian. OID subspaces are spanned by these modes, leading to optimal data compression tailored for purposes of observer and control design. A draft version of OID was introduced as the 'most observable decomposition' (MOD) in preliminary considerations (Jordan et al. 2007; Schlegel et al. 2009). OID is based solely on either: (i) empirical data representing both the hydrodynamic attractor and the observable; or (ii) only one of these quantities, presupposing that the other quantity can be provided using a known analytical relationship of hydrodynamics and observable. OID is applicable to a wide class of structure identification problems, assuming that the coherent dynamics of the observable is captured by a linear mapping from the hydrodynamics to the fluctuations of the considered observable.

As a first demonstration of its dimension reduction capability, OID is applied to distil the flow velocity structures most related to the lift force and to the drag force fluctuation. Because the OID modes can be compared with well-known 


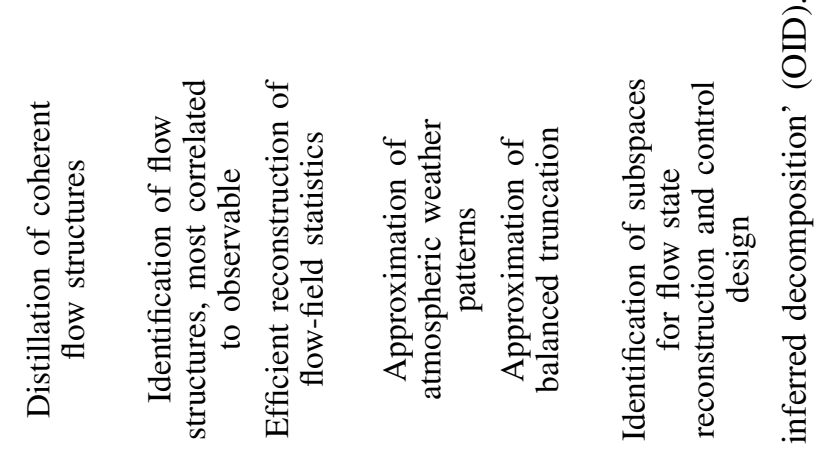

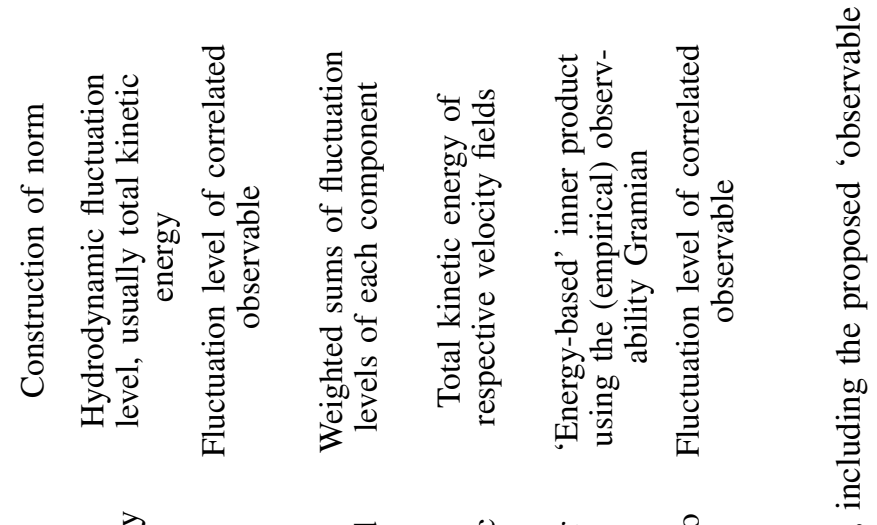

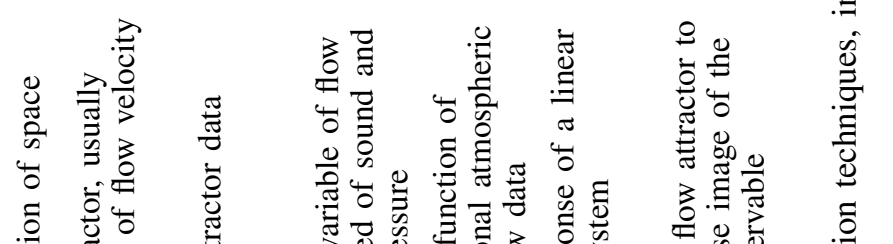

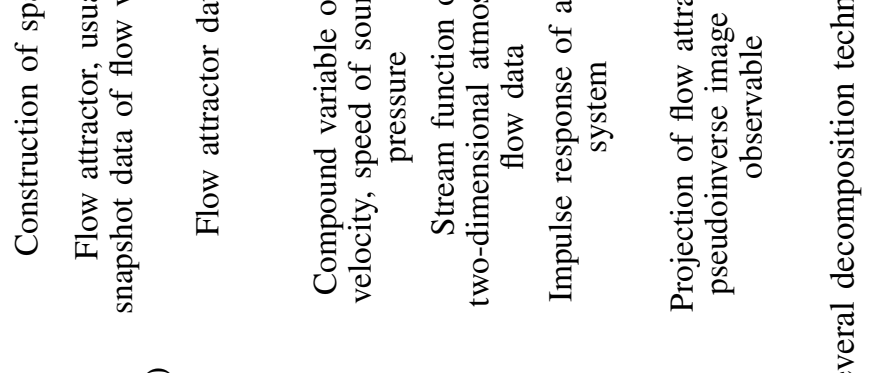

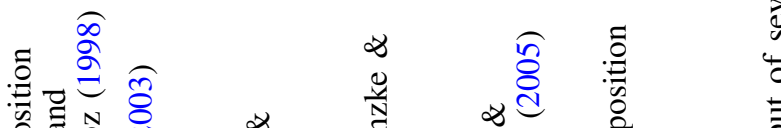

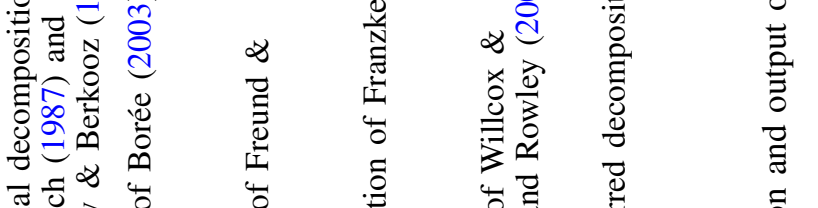

चَ.

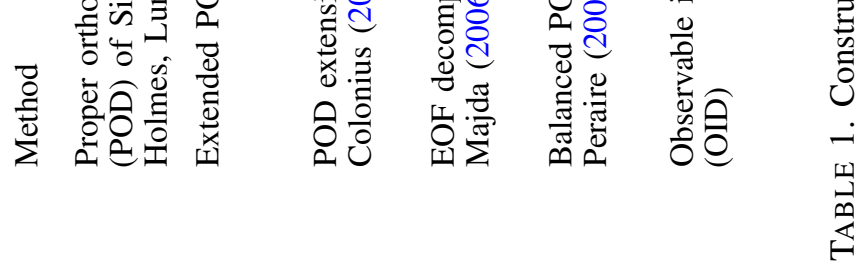


force-related structures (Protas \& Wesfreid 2003; Bergmann, Cordier \& Brancher 2005), this constitutes an exercise of a first check of OID's physical plausibility.

A major goal of the modelling efforts of this paper is to provide a physical understanding of shear flow noise generation. The need for such a physical understanding is motivated by ongoing efforts from the beginning of civil air traffic with jet engines to suppress jet noise from engine exhausts leading to larger bypass ratios of the jet engine, geometrical modifications of the nozzle trailing edge and active control devices like plasma actuators, microjets, fluidic chevrons and for acoustic forcing (see e.g. reviews in Tam 1998; Samimy et al. 2007; Jordan \& Gervais 2008; Laurendeau et al. 2008). Yet an intuitive understanding of the noiseproducing structures is still in its infancy after more than five decades of jet noise research (see e.g. Panda, Seasholtz \& Elam 2005). The complexity of this problem can be ascribed to the high dimensionality and the broadband spectrum of the flow state attractor. Presently, the main theoretical handle on noise source mechanisms in turbulent shear flows is given by the acoustic analogy, that of Lighthill (1952) being the most straightforward. The production of shear flow noise can be understood as a matching of scales between a 'source' term constructed from the flow field and an acoustic medium loosely thought of as the irrotational region surrounding the flow. By means of this scale matching (known as acoustic matching), a one-way transmission of propagative energy is established between the flow and the aeroacoustic far field. Here, only a very small part of the turbulence energy is transformed into energy of the aeroacoustic far field by a subtle evolution of turbulent structures and their interactions (Ffowcs Williams 1963; Crighton 1975). For subsonic jet flows, typical system dimensions of a few hundred modes of the most energy-efficient POD are obtained (see e.g. Gröschel et al. 2007). However, as a first hint towards loworder representations, it is moreover shown in Freund \& Colonius (2002, 2009) that representations of significantly lower order are realizable using the coherent part of the jet pressure field. As will be seen later, such considerations provide key enablers of the goal-oriented OID approach to pursue a significant dimension reduction. Preliminary results are indeed encouraging (Jordan et al. 2007).

The paper is organized as follows. Starting from the well-known POD and EPOD approaches, the principles of OID as an empirical structure identification method are outlined in $\S 2$. In $\S 3$, OID is applied to a cylinder wake flow where the observable is represented by lift and by drag fluctuation, respectively. To obtain a physical understanding of the noise generation in shear flows, OID results are presented for aeroacoustic far-field observables of a two-dimensional mixing layer and a three-dimensional $M a=0.9$ jet in $\$ \S 4$ and 5, respectively. In the Appendix, further mathematical details of the OID variants and the filtering of OID structures are specified.

\section{Snapshot-based flow decomposition methods}

In this section, reduced-order representations of the fluctuations (i.e. perturbations of a mean state $\langle\boldsymbol{u}\rangle$, e.g. the time average) of a given hydrodynamic quantity $\boldsymbol{u}$ are proposed by empirical Galerkin approximations,

$$
\boldsymbol{u}^{\prime}(\boldsymbol{x}, t):=\boldsymbol{u}-\langle\boldsymbol{u}\rangle \approx \sum_{i=1}^{L} a_{i}^{A}(t) \boldsymbol{u}_{i}^{A}(\boldsymbol{x}),
$$

to perform an optimal flow resolution of a given observable $\boldsymbol{q}$, which is linearly related to the hydrodynamic quantity. The decomposition is based on $L$ space-dependent 
modes $\boldsymbol{u}_{i}^{A}$, which have to be determined, and corresponding time-dependent mode coefficients $a_{i}^{A}$. In the following, we consider the flow velocity as hydrodynamic quantity, and aeroacoustic or aerodynamic observables. In a more abstract perspective, all of the subsequent considerations can be applied straightforwardly to arbitrary physical quantities.

Starting from the POD of the hydrodynamic attractor and of the observable in $\S 2.1$, the known extended POD (EPOD) approach is revisited in $\S 2.2$, leading to a first decomposition of the class (2.1). EPOD is set in $\S 2.3$ in a mathematically rigorous framework for definition of POD generalizations. Using this framework, a further POD generalization is derived in $\$ 2.4$ by employing the well-known Moore-Penrose pseudoinverse. Thus, the 'observable inferred decomposition' is proposed in $\S 2.5$. In this subsection, a variation of Sirovich's POD snapshot method is provided for computation of OID. Finally, the treatment and implementation of time delays is discussed in $\S 2.6$.

\subsection{Proper orthogonal decomposition (POD)}

Commonly in POD, velocity fluctuations are decomposed by the linear expansion into $N$ spatial POD modes $\boldsymbol{u}_{i}(\boldsymbol{x})$,

$$
\boldsymbol{u}^{\prime}(\boldsymbol{x}, t) \approx \sum_{i=1}^{N} a_{i}(t) \boldsymbol{u}_{i}(\boldsymbol{x}),
$$

using their mode coefficients $a_{i}(t):=\left(\boldsymbol{u}_{i}, \boldsymbol{u}^{\prime}\right)_{\Omega}$, defined via the inner product $(\cdot, \cdot)_{\Omega}$ of the function space $\boldsymbol{S}^{u} \subseteq \boldsymbol{L}^{2}(\Omega)$ of the hydrodynamic attractor. POD decomposes the flow velocity most efficiently for the resolution of

$$
Q^{\Omega}\left(\boldsymbol{u}^{\prime}\right):=\left\langle\int_{\Omega} \boldsymbol{u}^{\prime} \cdot \boldsymbol{u}^{\prime} \mathrm{d} \boldsymbol{x}\right\rangle=\left\langle\left(\boldsymbol{u}^{\prime}, \boldsymbol{u}^{\prime}\right)_{\Omega}\right\rangle,
$$

a goal functional representing twice the total kinetic fluctuation energy $\frac{1}{2} Q^{\Omega}\left(\boldsymbol{u}^{\prime}\right)$. This optimal resolution differs from the targeted flow resolution of the observable by the decomposition (2.1). Optimal resolution here means that the error $Q^{\Omega}\left(\boldsymbol{r}_{i}\right)$ of the residual $\boldsymbol{r}_{i}:=\boldsymbol{u}^{\prime}-\left(\boldsymbol{u}^{\prime}, \boldsymbol{u}_{1}\right)_{\Omega} \boldsymbol{u}_{1}-\cdots-\left(\boldsymbol{u}^{\prime}, \boldsymbol{u}_{i}\right)_{\Omega} \boldsymbol{u}_{i}$ is minimized for each $i=1, \ldots, N$. The modally resolved total kinetic energy is quantified by half of the respective POD eigenvalue $\lambda_{i}^{u}=\left\langle\left(\boldsymbol{u}_{i}, \boldsymbol{u}^{\prime}\right)_{\Omega}^{2}\right\rangle=\left\langle a_{i}^{2}\right\rangle$.

The expansion (2.2) is generalized for an arbitrary observable $\boldsymbol{q}$ (e.g. a sensor field of aeroacoustic pressure) via

$$
\boldsymbol{q}^{\prime}(\boldsymbol{y}, t) \approx \sum_{i=1}^{M} b_{i}(t) \boldsymbol{q}_{i}(\boldsymbol{y}) .
$$

Analogously, the POD of the observable can be considered to decompose the fluctuations $\boldsymbol{q}^{\prime}$ most efficiently for the resolution of the fluctuation level $Q^{\Gamma}\left(\boldsymbol{q}^{\prime}\right)$ (e.g. noise level of an aeroacoustic observable) of the observable $\boldsymbol{q}=\boldsymbol{q}(\boldsymbol{y}, t)$, where the goal functional $Q^{\Gamma}(\boldsymbol{q})$ is defined via

$$
Q^{\Gamma}\left(\boldsymbol{q}^{\prime}\right):=\left\langle\int_{\Gamma} \boldsymbol{q}^{\prime} \cdot \boldsymbol{q}^{\prime} \mathrm{d} \boldsymbol{y}\right\rangle=\left\langle\left(\boldsymbol{q}^{\prime}, \boldsymbol{q}^{\prime}\right)_{\Gamma}\right\rangle
$$

using the inner product $(\cdot, \cdot)_{\Gamma}$ of the function space $\boldsymbol{S}^{q} \subseteq \boldsymbol{L}^{2}(\Gamma)$ of the observable. Note that the domain $\Gamma$ of the observable may be distinct from the domain $\Omega$ of 
the considered flow region. Again, the resolution by each mode $\boldsymbol{q}_{i}$ is measured by the respective POD eigenvalue $\lambda_{i}^{q}=\left\langle\left(\boldsymbol{q}_{i}, \boldsymbol{q}^{\prime}\right)_{\Gamma}^{2}\right\rangle=\left\langle b_{i}^{2}\right\rangle$.

In the POD approach, the most efficiently resolved goal functional is thus determined by the fluctuation level of the decomposed field and cannot be chosen independently from this field. This inflexibility adversely affects POD's capability for reduced-order modelling and control: a large number of dynamical degrees of freedom might be required to capture the most important flow events for the generation of a considered aerodynamic or aeroacoustic observable, if only a small part of the hydrodynamic fluctuation level contributes to the generation of the observable! By way of example, for the free shear flow investigation in this paper, only a small part of the total kinetic energy is transformed into acoustic energy (see $\S \S 4$ and 5).

However, when the focus is on the manipulation only of the coherent flow part, representations (2.2) and (2.4) may act as prefilters with $N$ and $M$ sufficiently large to capture the considered physical processes for flow control. Thus, the vectors

$$
\begin{aligned}
& \boldsymbol{a}(t):=\left[a_{1}(t), a_{2}(t), \ldots, a_{N}(t)\right]^{\mathrm{T}}, \\
& \boldsymbol{b}(t):=\left[b_{1}(t), b_{2}(t), \ldots, b_{M}(t)\right]^{\mathrm{T}},
\end{aligned}
$$

of the respective POD mode coefficients are considered instead of the hydrodynamic field $\boldsymbol{u}(\boldsymbol{x}, t)$ and the observable $\boldsymbol{q}(\boldsymbol{y}, t)$. Respectively, for the Euclidean vector spaces $S^{a} \subseteq \mathbb{R}^{N}$ and $\boldsymbol{S}^{b} \subseteq \mathbb{R}^{M}$ of the POD mode coefficients, the goal functionals $Q^{\Omega}\left(\boldsymbol{u}^{\prime}\right)$ and $Q^{\Gamma}\left(\boldsymbol{q}^{\prime}\right)$ are approximated by $Q^{E}(\boldsymbol{a})$ and $Q^{E}(\boldsymbol{b})$, defined via

$$
Q^{E}(\boldsymbol{a}):=\langle\boldsymbol{a} \cdot \boldsymbol{a}\rangle, \quad Q^{E}(\boldsymbol{b}):=\langle\boldsymbol{b} \cdot \boldsymbol{b}\rangle,
$$

where the Euclidean vector dot product ' $\because$ ' is employed. Although in general the dimensions $N$ of $\boldsymbol{a}$ and $M$ of $\boldsymbol{b}$ are not equal, the symbol $Q^{E}$ is used in both cases for simplicity. By application of the representations (2.2) and (2.4), note that POD results can be obtained by formal application of the POD algorithm to the coefficients $\boldsymbol{a}(t)$ and $\boldsymbol{b}(t)$ with the Euclidean vector dot product as inner product.

\subsection{Extended proper orthogonal decomposition (EPOD)}

The essential idea of the EPOD approach is explained in two steps, using the representations (2.2) and (2.4) of the previous subsection (see Picard \& Delville 2000; Maurel et al. 2001; Borée 2003).

Firstly, POD is generalized by the modification of the inner product considering the coherent parts of hydrodynamic attractor and observable. In the space of the POD mode coefficients, the inner vector product $(\boldsymbol{v}, \boldsymbol{w}):=\boldsymbol{v} \cdot \boldsymbol{w}$ is varied based on a linear stochastic estimation (LSE)

$$
b=c a .
$$

The modified inner product is given by $(\boldsymbol{v}, \boldsymbol{w})_{A}:=\boldsymbol{C} \boldsymbol{v} \cdot \boldsymbol{C} \boldsymbol{w}$, which constitutes an inner vector product on each linear subspace of $\boldsymbol{S}^{a}$, in which no non-zero vector of the null space of $\boldsymbol{C}$ is contained. Thus, in EPOD the optimal resolution of the 'correlated' goal functional

$$
Q^{A}(\boldsymbol{a}):=\langle\boldsymbol{C a} \cdot \boldsymbol{C a}\rangle
$$

is required. Note that $Q^{A}(\boldsymbol{a})$ is equal to $Q^{E}(\boldsymbol{b})$ by virtue of (2.8).

Secondly, the EPOD subspace spanned by the EPOD modes is defined to be the only part of the hydrodynamic fluctuations that is correlated to the fluctuations of the observable. Owing to this choice, arbitrariness of the definition of EPOD modes $\boldsymbol{u}_{i}^{A}$ 
for $M<N$ (i.e. $\boldsymbol{C}$ is a singular matrix with a continuum of pseudoinverses) is removed, which are defined via

$$
\boldsymbol{u}_{i}^{A}(\boldsymbol{x}):=\sum_{j=1}^{N} a_{i, j}^{u} \boldsymbol{u}_{J}(\boldsymbol{x}),
$$

based on the constant vectors $\boldsymbol{a}_{i}^{u}$, the POD vector obtained via application of the POD algorithm in the coefficient spaces with above changed inner product.

Thus, the directions of the hydrodynamic attractor are identified via EPOD, decomposing the coherent fluctuations most efficiently for the resolution of the correlated observable. Moreover, from given measurements of the observable, the most correlated and therefore most probable state of the hydrodynamic attractor is reconstructed.

\subsection{A unifying framework for POD generalization}

To design generalizations of POD by the modification of inner products, it is assumed that the relationship between the hydrodynamics and the observable is well approximated by a linear mapping. Generalizing the relationship (2.8), a propagation process is modelled via

$$
\boldsymbol{q}^{\prime}(\boldsymbol{y}, t+\tau)=\int_{\Omega} \boldsymbol{C}(\boldsymbol{x}, \boldsymbol{y}, \tau) \boldsymbol{u}^{\prime}(\boldsymbol{x}, t) \mathrm{d} \boldsymbol{x},
$$

based on a linear propagator $\boldsymbol{C}(\boldsymbol{x}, \boldsymbol{y}, \tau)$ that is dependent on the physical or fitted time delay $\tau$ of propagation and the spatial variables.

The linear relationship is rewritten in operator notation as

$$
\boldsymbol{q}^{\prime}(t+\tau)=\boldsymbol{C}_{A} \boldsymbol{u}^{\prime}(t)
$$

where $\boldsymbol{q}^{\prime}(t+\tau)$ and $\boldsymbol{u}^{\prime}(t)$ both represent the respective spatial fields at any given time. The operator $\boldsymbol{C}_{A}$ may be dependent only on the time delay $\tau$ of the physical propagation process, e.g. the aeroacoustic propagation. For reasons of simplicity, the time delay is set to zero in the following. Its implementation will be revisited in $\$ 2.6$.

Assumption (2.12), which we term the 'OID assumption', is true in general for small fluctuations. At larger amplitudes, the existence of a meaningful linear mapping $\boldsymbol{C}_{A}$ has to be verified for each configuration. For the configurations employed in subsequent sections, this assumption is well founded for the considered flow configurations and goal functionals, because the generation of the observables by the hydrodynamics can be traced back mainly to a linear mechanism that can be identified by correlating these two fields. The OID assumption is violated for a strong nonlinear dependence of the observable on the hydrodynamics, like, for example, the consideration of self-noise (see $\S 5$ ), originating in the acoustic source term as the observable and the velocity fluctuations as the hydrodynamic quantity. To exclude any dependence of the observable on quantities other than the hydrodynamic quantity, $\boldsymbol{C}_{A}$ is furthermore assumed to represent a surjective mapping from the function space of the hydrodynamic attractor, denoted by $\boldsymbol{S}^{u}$, to the function space of the observable, denoted by $\boldsymbol{S}^{q}$. Moreover, we consider only the non-trivial case $\operatorname{dim} \boldsymbol{S}^{q}<\operatorname{dim} \boldsymbol{S}^{u}$, that is, $M<N$ in terms of the POD representations (2.2) and (2.4). In this case $\boldsymbol{C}$ is a singular matrix.

Like in the EPOD approach, the hydrodynamic field is decomposed by the flow representation (2.1) most efficiently for the resolution of the correlated goal functional

$$
Q^{A}\left(\boldsymbol{u}^{\prime}\right):=\left\langle\left(\boldsymbol{C}_{A} \boldsymbol{u}^{\prime}, \boldsymbol{C}_{A} \boldsymbol{u}^{\prime}\right)_{\Gamma}\right\rangle=Q^{\Gamma}\left(\boldsymbol{C}_{A} \boldsymbol{u}^{\prime}\right)=Q^{\Gamma}\left(\boldsymbol{q}^{\prime}\right)
$$


based on the linear mapping $\boldsymbol{C}_{A}$. The correlated goal functional $Q^{A}\left(\boldsymbol{u}^{\prime}\right)$ is equal to $Q^{\Gamma}\left(\boldsymbol{q}^{\prime}\right)$ (at least in a good approximation), as ensured via the OID assumption (2.12). An inner product is defined in a suitable hydrodynamic subspace by the product $\left(\boldsymbol{C}_{A} \boldsymbol{f}, \boldsymbol{C}_{A} \boldsymbol{g}\right)_{\Gamma}$ with hydrodynamic fields $\boldsymbol{f}$ and $\boldsymbol{g}$. Note that POD represents the special case of this approach with identical fluctuation fields of hydrodynamics and observable, i.e. if $\boldsymbol{C}_{A}$ coincides with the identity map.

As a first approach, the desired modes $\boldsymbol{u}_{i}^{A}$, decomposing the hydrodynamic attractor most efficiently for the resolution of the correlated goal functional $Q^{A}\left(\boldsymbol{u}^{\prime}\right)$, are extracted from the POD modes of the observable using an inversion of the linear relationship (2.12),

$$
\boldsymbol{u}_{i}^{A}:=\boldsymbol{C}_{A}^{-} \boldsymbol{q}_{i}
$$

The concept of the pseudoinverse $\boldsymbol{C}_{A}^{-}$of an operator represents a straightforward generalization of the pseudoinverse of a matrix (see Ben-Israel \& Greville 2003). We term a linear operator $\boldsymbol{C}_{A}^{-}$(or matrix $\boldsymbol{C}^{-}$) a 'pseudoinverse' of the operator $\boldsymbol{C}_{A}$ (or matrix $\boldsymbol{C}$ ) if the equations $\boldsymbol{C}_{A} \boldsymbol{C}_{A}^{-} \boldsymbol{C}_{A}=\boldsymbol{C}_{A}$ and $\boldsymbol{C}_{A}^{-} \boldsymbol{C}_{A} \boldsymbol{C}_{A}^{-}=\boldsymbol{C}_{A}^{-} \quad$ (or $\boldsymbol{C} \boldsymbol{C}^{-} \boldsymbol{C}=\boldsymbol{C}$ and $\boldsymbol{C}^{-} \boldsymbol{C}^{-}=\boldsymbol{C}^{-}$) are fulfilled. In the case that a unique inverse exist, the only pseudoinverse is given by this inverse.

The desired optimal resolution of $Q^{A}\left(\boldsymbol{u}^{\prime}\right)$ is proven by application of $\boldsymbol{C}_{A}$ to the modes $\boldsymbol{u}_{i}^{A}$. These modes are mapped to the POD modes $\boldsymbol{C}_{A} \boldsymbol{u}_{i}^{A}=\boldsymbol{q}_{i}$. Here, the fact is utilized that $\boldsymbol{C}_{A} \boldsymbol{C}_{A}^{-}$coincides with the identity map because $\boldsymbol{C}_{A}$ is surjective. Thus, the optimal resolution of $Q^{\Gamma}\left(\boldsymbol{q}^{\prime}\right)$ by the POD modes $\boldsymbol{q}_{i}$ of the observable is transferred to the optimal resolution of $Q^{A}\left(\boldsymbol{u}^{\prime}\right)$ by the modes $\boldsymbol{u}_{i}^{A}$. Thus these modes are sorted by the resolved level of the correlated goal functional $Q^{A}(\boldsymbol{u})$ from largest to smallest, quantified by the respective POD eigenvalues $\lambda_{i}^{q}=Q^{\Gamma}\left(b_{i} \boldsymbol{q}_{i}\right)=Q^{\Gamma}\left(a_{i}^{A} \boldsymbol{C}_{A} \boldsymbol{u}_{i}^{A}\right)$ of the POD analysis of the observable (see Holmes et al. 1998, and the Appendix). Orthonormality of the modes $\boldsymbol{u}_{i}^{A}$ is ensured in the sense of the modified inner product, i.e. $\left(\boldsymbol{C}_{A} \boldsymbol{u}_{i}^{A}, \boldsymbol{C}_{A} \boldsymbol{u}_{j}^{A}\right)_{\Gamma}=1$ for $i=j$, and zero otherwise, but not for the common POD inner product $(\cdot, \cdot)_{\Omega}$.

Using the POD representations (2.2) and (2.4), this methodology can be completely described in the finite-dimensional spaces of the POD mode coefficients $\boldsymbol{a}$ and $\boldsymbol{b}$. First the matrix $\boldsymbol{C}$ of the linear relationship (2.8) is identified using LSE or directly from the operator $\boldsymbol{C}_{A}$, if the relationship (2.12) is analytically known. As POD modes, the unit vectors $\boldsymbol{e}_{i}$ are obtained from a POD analysis of the vector-valued dynamics $\boldsymbol{b}(t)$ using the Euclidean vector product as inner product. The modes $\boldsymbol{u}_{i}^{A}$ are obtained from application of the pseudoinverse $\boldsymbol{C}^{-}$of $\boldsymbol{C}$ onto the POD modes of the observable,

$$
\boldsymbol{a}_{i}^{u}:=\boldsymbol{C}^{-} \boldsymbol{e}_{i},
$$

and (2.10), where the vectors $\boldsymbol{a}_{i}^{u}$ decompose the POD coefficient vector $\boldsymbol{a}$ most optimal for the resolution of $Q^{A}(\boldsymbol{a})$ defined in (2.9). Thus, the $\boldsymbol{u}_{i}^{A}$ modes are one-to-one related to the columns of $\boldsymbol{C}^{-}$.

The pseudoinverse matrix $\boldsymbol{C}^{-}$is not uniquely defined for the considered case $M<N$. Thus, the vectors $\boldsymbol{a}_{i}^{u}$ and therefore the modes $\boldsymbol{u}_{i}^{A}$ are at first not well defined via the above definitions, as expounded in the subsequent example.

EXAMPLE 2.1. Let the hydrodynamic data ensemble be represented by the following harmonic oscillator and an observable (one-dimensional) by the sine signal,

$$
\boldsymbol{a}=\left[\begin{array}{l}
\sin (2 \pi t) \\
\cos (2 \pi t)
\end{array}\right], \quad b=\sin (2 \pi t),
$$


for all $t \in \mathbb{R}$. Thus, $Q^{E}(b)=1 / 2$. The linear mapping from the hydrodynamic field to the observable is given by the projection $\boldsymbol{C}=[1,0]$ onto the first component of $\boldsymbol{a}$. The goal functional $Q^{A}(\boldsymbol{a})=Q^{E}(b)$ is completely resolved by only one direction, e.g. by $\boldsymbol{a}^{u}=[1,0]^{\mathrm{T}}$. In contrast, two orthogonal directions of the hydrodynamic field are required to resolve $100 \%$ of $Q^{E}(\boldsymbol{a})$. However, $\boldsymbol{a}^{u}$ is not uniquely defined owing to the non-invertibility of $\boldsymbol{C}$; the complete resolution of $Q^{A}(\boldsymbol{a})$ is performed as well by any direction $\boldsymbol{a}^{u}=[\alpha, \beta]^{\mathrm{T}}$ with $\alpha \neq 0$.

\subsection{Application of the Moore-Penrose pseudoinverse}

In the case of EPOD modes, the pseudoinverse $\boldsymbol{C}^{-}$is tailored to observer design, because the EPOD space resolves the only part of the hydrodynamic field, correlated to the observable. Besides the assumptions of the previous subsections, it is therefore presupposed that the dynamics both of the hydrodynamic field and the observable are provided.

For the least-biased choice of a pseudoinverse, only measurements of the observable and the null space of the linear relationship (2.12) have to be known. No additional information is required, in contrast to EPOD employing the statistics of the hydrodynamic attractor. This choice is given by the well-known Moore-Penrose pseudoinverse, which can be defined by the following optimal property: for each observable $\boldsymbol{q}(t)$, the norm of $\boldsymbol{C}_{A}^{-} \boldsymbol{q}(t)$ at each time $t$ is minimized, i.e. the total kinetic energy $\frac{1}{2} Q^{\Omega}\left(\boldsymbol{u}^{\prime}\right)$ contained in the subspace spanned by the respective modes $\boldsymbol{u}_{i}^{A}$ is minimal for a given fluctuation level of the observable $Q^{\Gamma}\left(\boldsymbol{q}^{\prime}\right)$. A manipulation of the dynamics that leads to a reduction of kinetic energy in this subspace therefore causes a reduction of fluctuation level of the observable. Thus, the use of the Moore-Penrose pseudoinverse is predestinated for Lyapunov control design, e.g. energy-based control design, to suppress the fluctuations of the observable.

\subsection{A generalized decomposition approach}

In summary of the previous subsections, a unifying framework for generalizations of POD has been provided using modified, observable-weighted inner products. The methodology of the resulting decomposition, which we term 'observable inferred decomposition' (OID), is outlined in figure 1. POD represents the special case of OID with identical fluctuation fields of hydrodynamics and observable, i.e. if $\boldsymbol{C}_{A}$ coincides with the identity map. The modes $\boldsymbol{u}_{i}^{A}$ and the vectors $\boldsymbol{a}_{i}^{u}$, the subspaces of the hydrodynamic space spanned by these modes, and the coefficients of the 'OID representation' (2.1) are termed 'OID modes', 'OID subspace' and 'OID coefficients', respectively. There are two types of pseudoinverse, defining two variants of OID, both given by a respective optimal property:

(a) By the 'least-residual principle', the error of the reconstruction of the hydrodynamic field is minimized via application of the pseudoinverse to the observable. Thus, the variant of the 'least-residual OID' (LR-OID) is provided. In the case that the POD representation (2.2) is used to prefilter coherent structures, this variant coincides with the EPOD approach. However, LR-OID is defined for a more general class of structure identification problems. Like in the EPOD approach, the most correlated (i.e. most probable) state of the hydrodynamic attractor can be reconstructed in the LR-OID subspace from given data of the observable, thus preprocessing efficient observer design.

(b) By the 'principle of least energy', the total kinetic energy is minimal in the OID subspace for a given fluctuation level fulfilled by the Moore-Penrose 


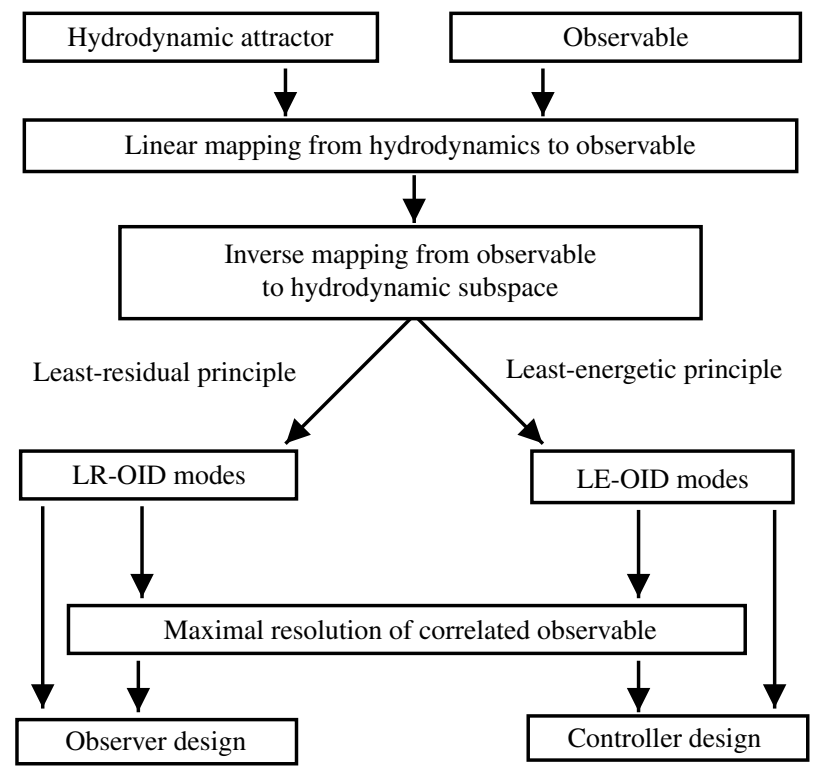

FIGURE 1. Principle of the observable inferred decomposition.

pseudoinverse. This defines the 'least-energetic OID' (LE-OID), which quantifies the smallest displacement in phase space that a controller has to perform for reduction of the goal functional to zero. Exploiting this definition, an energybased control strategy to suppress the fluctuations of the observable is to pursue the reduction of the total kinetic energy in the LE-OID subspace, which is by definition irreducible with respect to maintaining the level of the correlated fluctuations of the observable.

More mathematically rigorous definitions of the LR- and LE-OID variants are detailed in the Appendix. The above terminologies are adapted to the OID variants, leading to the terms 'LR-OID modes', 'LE-OID modes', 'LR-OID coefficients', 'LE-OID coefficients', etc.

For computation of OID, here an analogue of Sirovich's POD snapshot method (Sirovich 1987) is provided. As empirical basis, the data are given as an ensemble of statistically independent snapshots $\left\{\boldsymbol{u}\left(t^{1}\right), \ldots, \boldsymbol{u}\left(t^{K}\right)\right\}$ of the hydrodynamic attractor and as an ensemble of statistically independent snapshots $\left\{\boldsymbol{q}\left(t^{1}\right), \ldots, \boldsymbol{q}\left(t^{K}\right)\right\}$. Here the number of snapshots is denoted by $K$. The times of the snapshots are denoted by $t^{1}, \ldots, t^{K}$. The following algorithm can be easily varied, if only one of these ensembles is given and linear relationship (2.12) is, for example, analytically known. The hydrodynamic fluctuations are denoted by $\boldsymbol{u}^{1}:=\boldsymbol{u}\left(t^{1}\right)-\langle\boldsymbol{u}\rangle, \ldots, \boldsymbol{u}^{K}:=\boldsymbol{u}\left(t^{K}\right)-\langle\boldsymbol{u}\rangle$, and the fluctuations of the observable by $\boldsymbol{q}^{1}:=\boldsymbol{q}\left(t^{1}\right)-\langle\boldsymbol{q}\rangle, \ldots, \boldsymbol{q}^{K}:=\boldsymbol{q}\left(t^{K}\right)-\langle\boldsymbol{q}\rangle$, where means are estimated by the (pointwise) arithmetic mean

$$
\langle\boldsymbol{u}\rangle=\frac{1}{K} \sum_{i=1}^{K} \boldsymbol{u}\left(t^{i}\right), \quad\langle\boldsymbol{q}\rangle=\frac{1}{K} \sum_{i=1}^{K} \boldsymbol{q}\left(t^{i}\right) .
$$

First of all, the POD representations (2.2) and (2.4) are computed by the POD snapshot method (see Sirovich 1987; Holmes et al. 1998, for details). Thereby, fluctuations of hydrodynamics and observable are completely described by the 


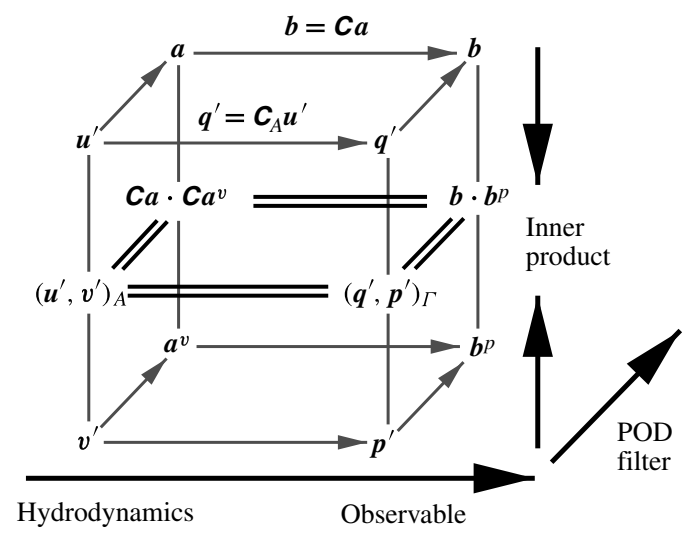

FIGURE 2. Commutative diagram of OID products, defined in the hydrodynamic state space, the space of the observable and the respective POD subspace representations.

respective vectors of POD mode coefficients $\boldsymbol{a}^{1}, \ldots, \boldsymbol{a}^{K}$ and $\boldsymbol{b}^{1}, \ldots, \boldsymbol{b}^{K}$ such that the dynamics of the coherent structures is represented by

$$
\boldsymbol{u}^{j}=\sum_{i=1}^{N} a_{i}^{j} \boldsymbol{u}_{i}, \quad \boldsymbol{q}^{j}=\sum_{i=1}^{M} b_{i}^{j} \boldsymbol{q}_{i},
$$

at each snapshot time $t^{j}, j=1, \ldots, K$. The number of utilized POD modes $M$ and $N$ is chosen such that $M \leqslant N<K-1$. Using the POD filter, the desired linear mapping $\boldsymbol{C}_{A}$ of (2.12) is approximated by its matrix-valued analogue $\boldsymbol{C}$ defined in (2.8), which can be computed by linear stochastic estimation.

In the next step, the OID snapshot matrix

$$
\boldsymbol{R}_{u}^{O I D}=\left[\frac{1}{K}\left(\boldsymbol{u}^{j}, \boldsymbol{u}^{k}\right)_{A}\right]_{j, k=1}^{K}
$$

has to be determined with

$$
\left(\boldsymbol{u}^{\prime}, \boldsymbol{v}^{\prime}\right)_{A}:=\left(\boldsymbol{C}_{A} \boldsymbol{u}^{\prime}, \boldsymbol{C}_{A} \boldsymbol{v}^{\prime}\right)_{\Gamma},
$$

approximated by

$$
\left(\boldsymbol{u}^{\prime}, \boldsymbol{v}^{\prime}\right)_{A} \approx \boldsymbol{C} \boldsymbol{a} \cdot \boldsymbol{C} \boldsymbol{a}^{v}=\sum_{i=1}^{M}\left(\sum_{j=1}^{N} C_{i j} a_{j}\right)\left(\sum_{j=1}^{N} C_{i j} a_{j}^{v}\right),
$$

where the vector of mode coefficients of $\boldsymbol{v}^{\prime}$ is denoted by $\boldsymbol{a}^{v}$, and the $C_{i j}$ are the matrix elements. The relations of the inner products defined for the hydrodynamics fields and the observable, respectively, in the function spaces and the finite-dimensional spaces of the POD coefficients are illustrated in figure 2, demonstrating that the OID snapshot method can be considered as a generalization of the POD snapshot method with new inner products.

The OID snapshot matrix can now be computed from this approximation via

$$
\boldsymbol{R}_{u}^{O I D}=\left[\frac{1}{K}\left(\boldsymbol{a}^{j}, \boldsymbol{a}^{k}\right)_{A}\right]_{j, k=1}^{K}=\frac{1}{K}\left[\boldsymbol{C} \boldsymbol{a}^{j} \cdot \boldsymbol{C} \boldsymbol{a}^{k}\right]_{j, k=1}^{K} .
$$


We assume the OID eigenvalues $\lambda_{i}^{p}$ of the OID snapshot matrix, which as mentioned above are equal to the POD eigenvalues of the POD of the observable, to be sorted by size, starting from the largest. The eigenvalues will be verified by solving the eigenvalue equation

$$
\boldsymbol{R}_{u}^{O I D} \boldsymbol{c}^{[i]}=\lambda_{i}^{p} \boldsymbol{c}^{[i]},
$$

where the eigenvector of the $i$ th eigenvalue $\lambda_{i}^{p}$ is denoted by $\boldsymbol{c}^{[i]}$.

The LR-OID modes are obtained from

$$
\boldsymbol{u}_{i}^{A}=\sum_{j=1}^{K} d_{j}^{[i]} \boldsymbol{u}^{j} \quad \text { where } \boldsymbol{d}^{[i]}:=\sum_{m=1}^{K} c_{m}^{[i]} \boldsymbol{a}\left(t^{m}\right),
$$

which results in a formula coinciding with the computation of EPOD modes (see Maurel et al. 2001).

To calculate the LE-OID modes, all vectors $\boldsymbol{d}^{[i]}$ are projected onto the subspace spanned by the row vectors of the matrix $\boldsymbol{C}$. Let $\hat{\boldsymbol{c}}^{l}=\left[C_{l 1}, \ldots, C_{l K}\right]^{\mathrm{T}}$ be the transposed $l$ th row vector of $\boldsymbol{C}$. Then the projection of $\boldsymbol{d}^{[i]}$ is given by

$$
\hat{\boldsymbol{d}}^{[i]}=\sum_{l=1}^{M} \frac{\boldsymbol{d}^{[i]} \cdot \hat{\boldsymbol{c}}^{l}}{\hat{\boldsymbol{c}}^{l} \cdot \hat{\boldsymbol{c}}^{l}} \hat{\boldsymbol{c}}^{l}
$$

The $i$ th LE-OID mode is obtained from (2.24) using the projected $\hat{\boldsymbol{d}}^{[i]}$ instead of $\boldsymbol{d}^{[i]}$.

The OID mode coefficients of LR- or LE-OID modes are uniquely determined after orthonormalization of the $\boldsymbol{d}^{[i]}$ or $\hat{\boldsymbol{d}}^{[i]}$ vector set using

$$
a_{i}^{A}(t)=\boldsymbol{a}(t) \cdot \boldsymbol{d}^{[i]} \quad \text { or } \quad a_{i}^{A}(t)=\boldsymbol{a}(t) \cdot \hat{\boldsymbol{d}}^{[i]},
$$

respectively.

\subsection{Implementation of time delays}

Throughout the previous subsections, an instantaneous dependence of the observable on the hydrodynamics is presupposed. A larger class of structure identification problems may be tackled, revisiting the occurrence of a unique time delay $\tau$ in the equations of the OID assumption (2.11) or (2.12). This includes a configuration where the uniqueness of a time delay $\tau \neq 0$ is analytically known, e.g. for the arrival of separated vortices downstream a certain distance from a van Kármán vortex street.

However, in the aeroacoustic problems considered in this paper, usually there is a continuum, or after discretization a large number, of locally dependent, physical time delays. By modelling of this ensemble of physical propagation times via the OID assumption with a fitted, unique propagation time $\tau$, at first a filter of the aeroacoustic effects is constituted. However, because of the strong wave character of the aeroacoustic waves in the far field of mixing layers and the jet, future and past events are captured in this filtering. An insensitivity of this filter against the variations of the physical, aeroacoustic propagation times is enabled by strong correlation of the current with future and past events. Therefore, for OID identification of 'loud' flow structures, aeroacoustic propagation is modelled via a unique time delay. This time delay is fitted by maximization of the OID resolution. Following the above arguments, only small distortions of the 'loud' OID flow structures against the local spatial structures responsible for flow noise generation are expected. The first efforts of 
the authors to vary the OID assumption to implement several, or even a continuum of, time delays are interesting, but go beyond the scope of this paper.

OID with a unique time delay $\tau \neq 0$ can be computed in complete analogy to the case $\tau=0$ treated in the OID snapshot method of the previous subsection. Here, as data source, an ensemble of statistically independent snapshots $\left\{\boldsymbol{q}\left(t^{1}+\tau\right), \ldots, \boldsymbol{q}\left(t^{K}+\tau\right)\right\}$ of the observable is given, which is shifted by time delay $\tau$ in comparison to the ensemble of the hydrodynamic data. Moreover, the vector-valued analogue (2.8) of (2.12) is given by

$$
\boldsymbol{b}(t+\tau):=\boldsymbol{C}(\tau) \boldsymbol{a}(t),
$$

such that $\boldsymbol{C}$ is identified as above using LSE, but is dependent on $\tau$.

\section{Lift and drag optimized OIDs of cylinder wake flow}

In this section, OID structures are identified that are most related to lift and to drag fluctuation of a two-dimensional cylinder wake flow. The Reynolds number is $R e=U D / v=100$, based on the cylinder diameter $D$ and the oncoming flow $U$. For the following empirical investigations, 570 velocity snapshots with an equidistant time step of 0.1 convective time units are provided by a finite element Navier-Stokes solver. Details of this solver are given in Morzyński (1987) and Afanasiev (2003).

The OID assumption (2.12) with $\tau=0$ is guaranteed by the definition of the observable lift and drag fluctuation, which at least in a good approximation depend linearly and instantaneously on the velocity fluctuations and its POD representations - see Gerhard et al. (2003), Noack et al. (2003), Protas \& Wesfreid (2003), Bergmann et al. (2005) and Luchtenburg et al. (2009) for results of POD analyses.

As a result of each of the two OIDs of lift and drag fluctuation, only one OID mode resolves approximately $100 \%$ of the respective quantity. The obtained OID modes represent mainly the first and the second flow harmonics (see Noack et al. 2003). This is shown in figure 3, where the axis of the streamwise direction is denoted by $x$ and the axis of the transverse direction by $y$. Strikingly, these results are consistent with the well-known empirical fact that the lift force consists only of contributions of the odd harmonics and the drag force fluctuation consists only of contributions of the even harmonics, which has been explained theoretically (see Protas \& Wesfreid 2003). Lift force and drag force fluctuations are most susceptible to variations of the amplitudes of the first odd and even POD modes, which energetically dominate higher odd and even POD modes, respectively (see e.g. Noack et al. 2003; Luchtenburg et al. 2009).

\section{Acoustically optimized OID of a mixing layer}

In this section, 'loud' structures of a two-dimensional mixing layer are distilled by application of OID, optimized for an aeroacoustic goal functional. The mixing layer configuration is sketched in figure 4. The goal functional of the mixing-layer noise is given by the sum of variances of 74 density sensors in the far-field region (see figure 4). For the following empirical analyses, an ensemble of 3691 snapshots of velocity and density is employed with an equidistant time step of $\Delta t=1.68 \delta_{\omega} / \Delta U$ (see caption of figure 4), provided by a direct numerical simulation. Details of the direct numerical simulation are given in Freund (2001) and Wei \& Freund (2006).

Physical evidence of the OID assumption (2.12) is confirmed from investigations of the annular mixing layer arising at the end of the potential core of jet flows. The predominant linearity of the relationship between the turbulent fluctuations and the far-field pressure is shown in this region (see Lee \& Ribner 1972; Scharton \& White 

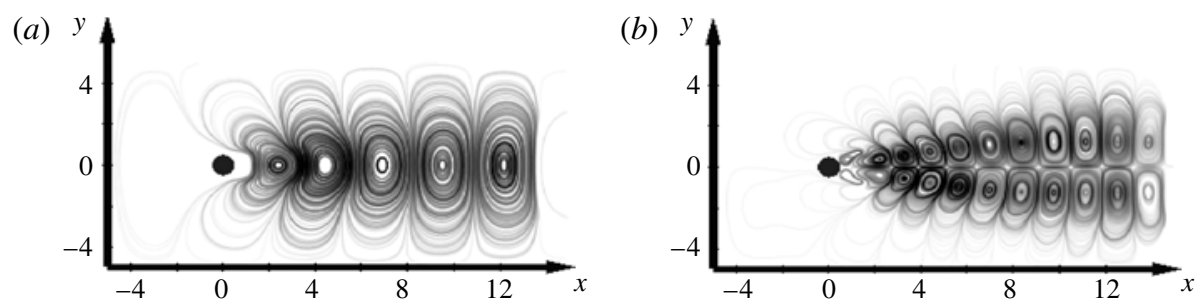

FIGURE 3. OID modes of a cylinder wake flow at $R e=100$. The OID modes resolve almost $100 \%$ of $(a)$ lift and $(b)$ drag fluctuations, respectively. In both panels, velocity streamlines are shown. The grid unit is given by the cylinder diameter. The OID variant is not indicated, because the results of LR-OID and of LE-OID coincide.

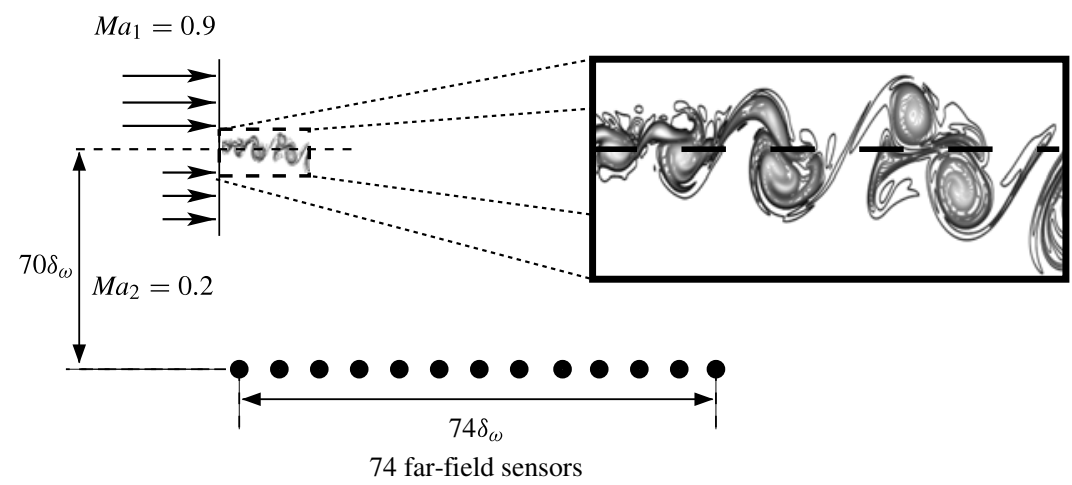

FIGURE 4. Sketch of the mixing-layer configuration at $R e_{u}=500$. The Reynolds number is defined by $R e_{u}=\rho_{\infty} \Delta U \delta_{\omega} / \mu$, employing the ambient density $\rho_{\infty}$ identical for both streams, the velocity difference $\Delta U$ across the layer, the inflow vorticity thickness $\delta_{\omega}=$ $\Delta U /|\mathrm{d} u / \mathrm{d} y|_{\max }$ of the initial hyperbolic tangent velocity profile and the constant viscosity $\mu$. The Mach numbers are given by $M a_{1}=U_{1} / c_{\infty}=0.9$ and $M a_{2}=U_{2} / c_{\infty}=0.2$, with the ambient speed of sound $a_{\infty}$. Further configuration parameters can be found in Wei (2004) and Wei \& Freund (2006). The velocity data are evaluated on a Cartesian grid in the domain $(x, y) \in\left[0 \delta_{\omega}, 100 \delta_{\omega}\right] \times\left[-20 \delta_{\omega}, 20 \delta_{\omega}\right]$, where the streamwise component is represented by the $x$ axis and the transverse component by the $y$ axis. The observable is represented by the density fluctuations, monitored by 74 density sensors. These sensors are equidistantly arranged on a linear array situated at $y=-70 \delta_{\omega}$ in the $M a=0.2$ stream and parallel to the $y=0$ axis.

1972; Seiner \& Reetoff 1974; Juvé, Sunyach \& Comte-Bellot 1980; Schaffar \& Hancy 1982), which is moreover identified to be the dominant source of jet noise.

As a first result of OID, an optimally fitted time delay $\tau$ is identified by the maximal OID resolution of the density fluctuations. As shown in figure 5, a single maximum of the OID resolution for identification of a fitted time delay of aeroacoustic propagation has been found. The nearly $90 \%$ correlation at this time delay corroborates the OID assumption (2.12).

Only four OID modes resolve $85 \%$ of the aeroacoustic far field. In comparison, the POD analyses of this flow and controlled counterparts extract a typical POD dimension of 20 for a resolution of $75 \%$ total kinetic energy as presented by Wei (2004) and Wei \& Freund (2006). Similar POD dimensions are obtained for threedimensional mixing layers as well (see Noack et al. 2005). In recent investigations, a 


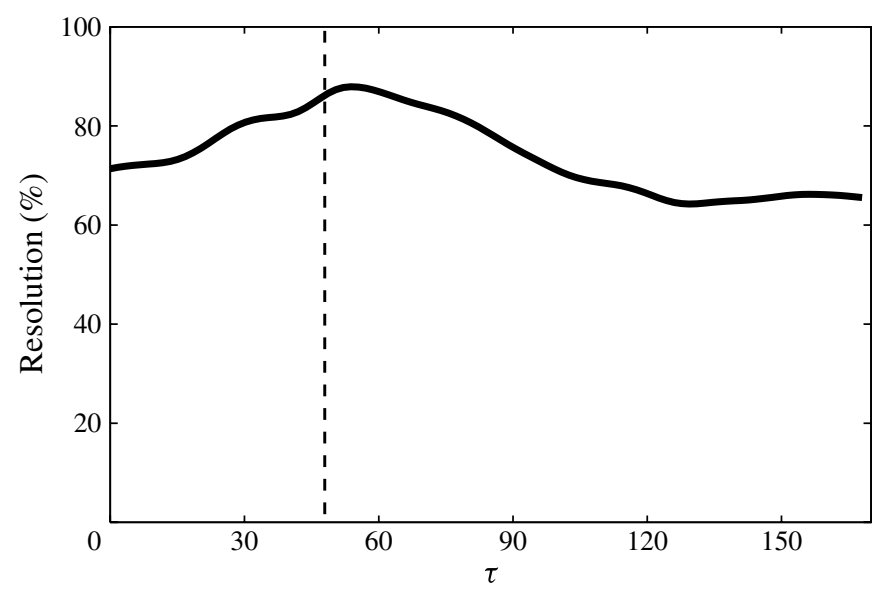

FIGURE 5. Percentage OID resolution of correlated noise over the propagation time delay $\tau$ between hydrodynamics and aeroacoustic sensor array (see (2.12)). The optimal propagation time is obtained by the maximum of $87.9 \%$ at $\tau=53.76 \delta_{\omega} / \Delta U$. The propagation time $\tau=49 \delta_{\omega} / \Delta U$, in which sound propagates along a distance of $70 \delta_{\omega}$, is represented by the vertical dashed line. The optimal propagation time is slightly larger due to sound propagation non-perpendicular to the jet axis. The non-vanishing resolution far from the maximum is ascribed to a dominant travelling wave character of the aeroacoustic observable. Thus, a significant long-term correlation of the observable is represented, where phase information of wave events is captured by a linear fit.

further dimension reduction is obtained using dynamic scaling of the modes and of the base flow (see Wei \& Rowley 2009).

POD, LR-OID and LE-OID modes are compared in figure 6 by their resolutions of correlated noise and total kinetic energy. As expected, the optimality of POD for the resolution of total kinetic energy and that of OID for the resolution of correlated noise are confirmed. More surprisingly, less than $0.1 \%$ total kinetic energy is resolved by the LE-OID modes, meaning that only a small portion of the total kinetic energy has to be manipulated for the purposes of noise control. In contrast, the amount of total kinetic energy reconstructible from LR-OID exceeds this value by two orders of magnitude.

The first four LR-OID modes are visualized in figure 7 and are reminiscent of noiseproducing events of vortex merging (see Jordan \& Gervais 2008) and of wavepackets that amplify and rapidly decay further downstream (see Crighton \& Huerre 1990). The respective LE-OID modes show significantly less coherence.

\section{Acoustically optimized OID of jet flow}

In this section, 'loud' structures of a three-dimensional, $M a=0.9$ jet are distilled by application of OID, optimized for a similar aeroacoustic goal functional as in the previous section. The jet configuration is sketched in figure 8. The Reynolds number $R e=U D / \nu=3600$ is based on the jet diameter $D$ and the inflow velocity $U$. The goal functional of jet noise is given from the sum of the variances of pressure sensors in the far field (see figure 8). For the following empirical analyses, an ensemble of 725 velocity snapshots is utilized with an equidistant time step of 0.2125 convective time units, provided by a large-eddy simulation (LES; see Meinke et al. 2002; Gröschel 

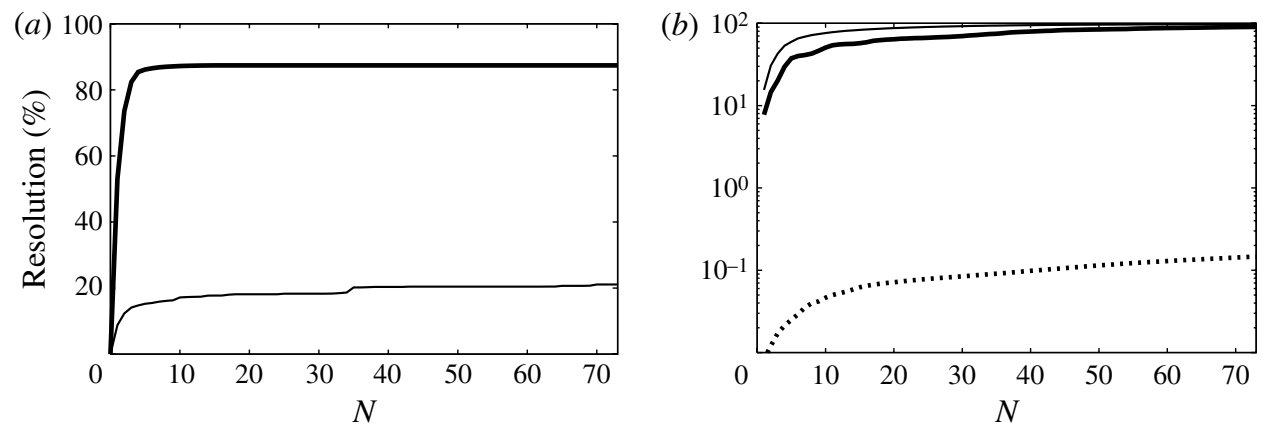

FIGURE 6. Percentage resolution of $(a)$ linearly correlated noise and $(b)$ total kinetic energy given by OID and POD modes, accumulated over the number of used modes represented by index $N$ on the $x$ axis. Curves related to LR-OID modes (thick full line), LE-OID modes (dotted line) and POD modes (thin full line) are displayed. In panel (a), the curves of LR-OID and LE-OID coincide.

et al. 2007). The aeroacoustic far-field data are computed from the LES data by a Ffowcs Williams-Hawkings solver for the $M a=0.9$ jet as described in Gröschel et al. (2008).

The physical validity of the OID assumption (2.12) is verified by known results: the fast pressure term (sometimes referred to as 'shear noise') has been shown to dominate in free jets in terms of the hydrodynamic, turbulent pressures, and to correlate better with the far-field pressure than the quadratic slow pressure ('self-noise') (see Lee \& Ribner 1972; Scharton \& White 1972; Seiner 1974; Seiner \& Reetoff 1974; Schaffar 1979; Juvé et al. 1980; Schaffar \& Hancy 1982; Panda et al. 2005). It has furthermore been demonstrated in Cavalieri et al. $(2011 a, b, c)$ that coherent flow structures generate noise by means of a wavepacket mechanism, while Rodriguez Alvarez et al. (2011) show how these wavepackets can be modelled in the framework of linear stability theory.

Moreover, a fitted time delay $\tau$ appropriate to (2.12) for modelling of the aeroacoustic propagation is identified as in the previous section by minimization of the OID residuum.

Employing OID, a reduction by one order of magnitude is achieved compared to the POD dimension (see figure 9). It can be seen that $90 \%$ of the correlated noise is resolved by only 24 OID modes! In contrast, POD analysis extracts a large number of dynamic degrees of freedom - more than 350 POD modes are needed to resolve more than $50 \%$ of the total kinetic energy (see Gröschel et al. 2007). In contrast, in figure 9 the resolved accumulated noise of POD modes, estimated by the linear mapping (2.12) from hydrodynamics to observable with fitted time delay $\tau$ instead of by physical propagation of an aeroacoustic analogy, indicates an overoptimization of the resolution. Similar POD results for this configuration have been found by Freund \& Colonius (2002).

In figure 10, the first six LR-OID modes and LE-OID modes are shown. Higher LR-OID or LE-OID modes reveal variously disorganized, smaller-scale activity. The first two LR-OID modes, resolving $48 \%$ of the correlated noise, identify asymmetric streaks in the region just downstream of the end of the potential core. These streaks contain noticeable helical structures. Cavalieri et al. (2011b) observed how such helical motions at the end of the potential core are important in increasing the acoustic efficiency of an axisymmetric wavepacket upstream of this region. The next LR-OID 
(a)

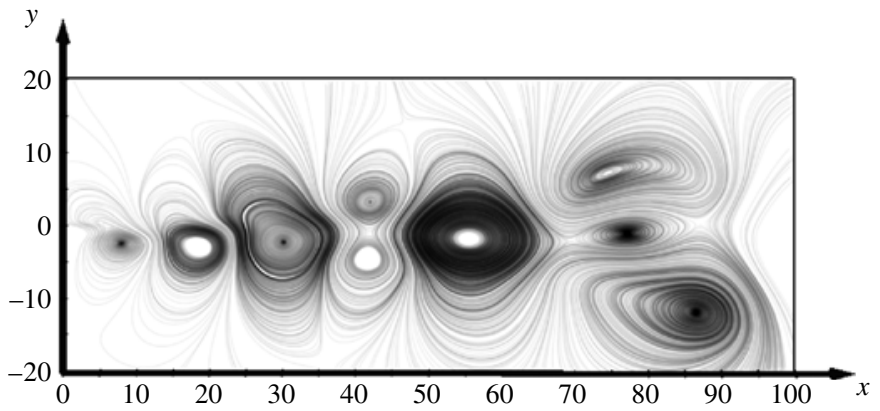

(b)

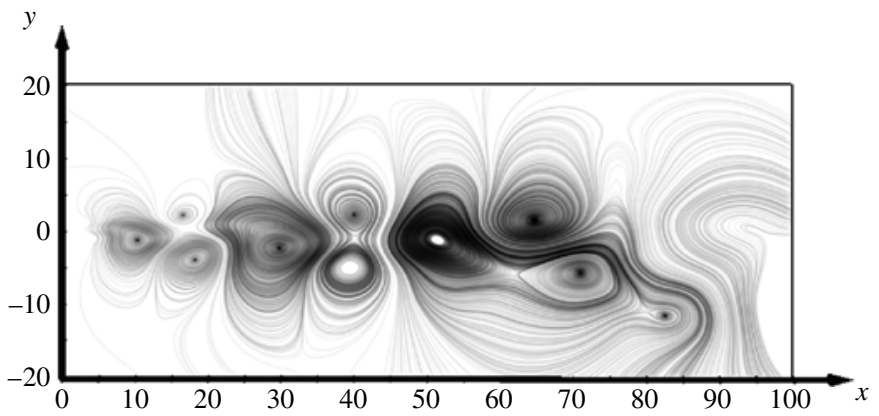

(c)

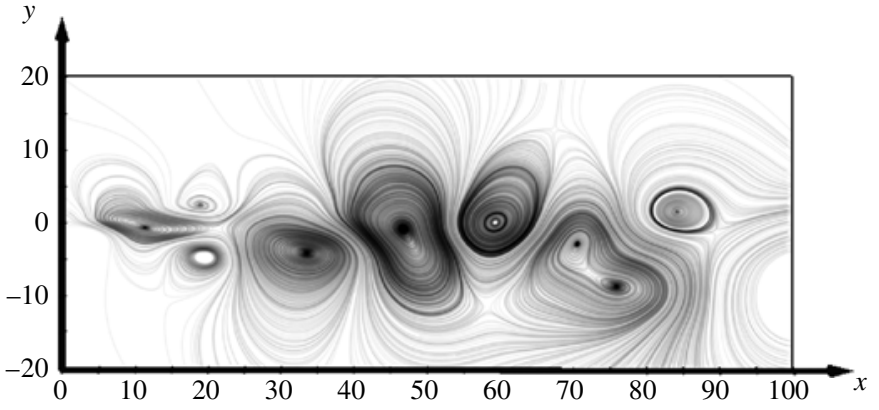

(d)

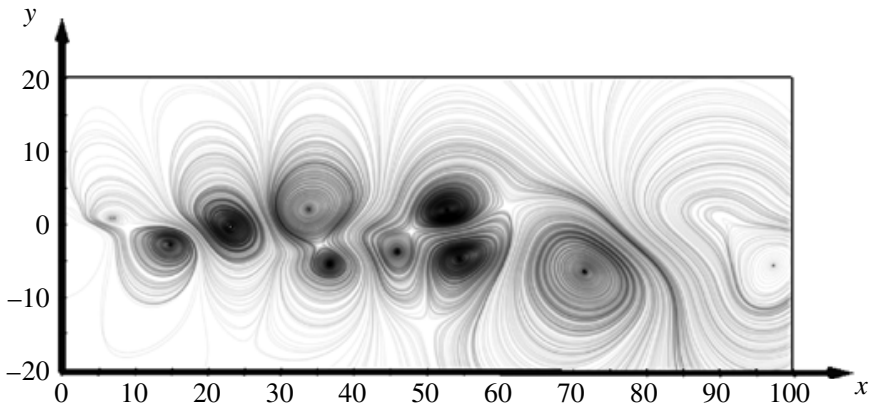

FIGURE 7. The first four LR-OID modes of the mixing layer, $i=1-4$ from $(a)$ to $(d)$, visualized by streamlines. The grid unit is given by the vorticity thickness $\delta_{\omega}$.

mode pair contributes $7 \%$ of the correlated noise. It shows structures comprising highly coherent, axisymmetric vortex-ring-like structures in the region upstream of the end of the potential core, which resemble the wavy structure of the radiating 


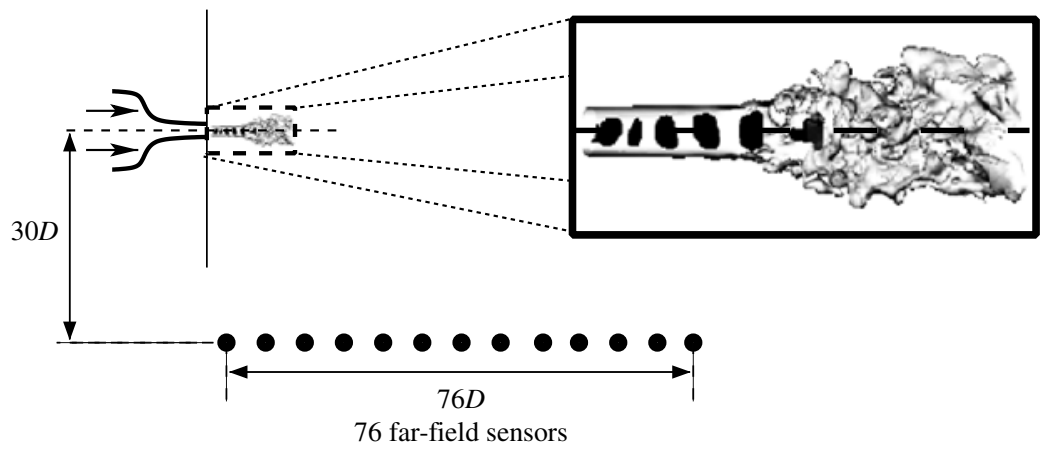

FIGURE 8. Sketch of the three-dimensional jet configuration at $R e_{D}=3600$ and $M a=0.9$. The velocity data are evaluated on a Cartesian grid in the domain $(x, y, z) \in[0 D, 14 D] \times$ $[-2.5 D, 2.5 D] \times[-2.5 D, 2.5 D]$, where again the streamwise direction is represented by the $x$ axis and transverse directions by the $y$ axis and the $z$ axis. The aeroacoustic observable is represented by 76 pressure sensors. These sensors are equidistantly arranged along a straight line $30 D$ away from the jet axis and parallel to it in the zero plane of the $z$ direction.

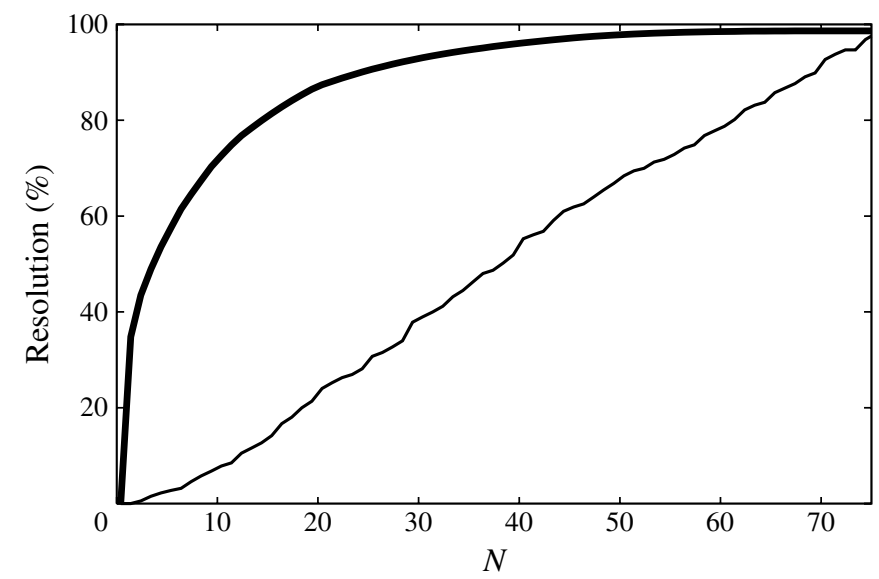

FIGURE 9. Percentage resolution of linearly correlated noise by OID and POD modes, accumulated over the number $N$ of used modes. Curves related to both types of OID modes (thick line) and to the POD modes (thin line) are displayed.

component of the Lighthill source term, as identified by Freund (2001), and the aforesaid axisymmetric wavepacket structures observed and modelled by Cavalieri et al. $(2011 a, b)$. The loud flow structures of both LR-OID mode pairs are in qualitative agreement with experiments (see e.g. Juvé et al. 1980; Guj, Carley \& Camussi 2003; Hileman et al. 2004; Coiffet et al. 2006). In figure $10(g-l)$, the first six LE-OID modes are shown. In comparison of the LE-OID modes with the LR-OID modes, the axisymmetric vortex rings vanish. Here helical structures become more dominant, corroborating the recent analysis of Freund \& Colonius (2009).

\section{Conclusions}

We propose a Galerkin expansion tailored towards a physical understanding of aerodynamic and aeroacoustic aspects of shear flows. By POD, the modal expansion 
(a)

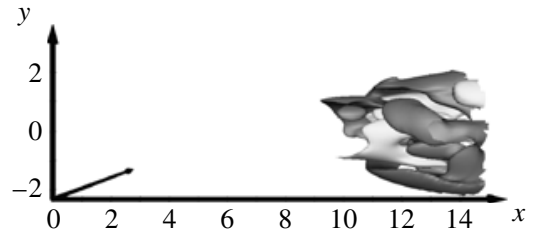

(b)

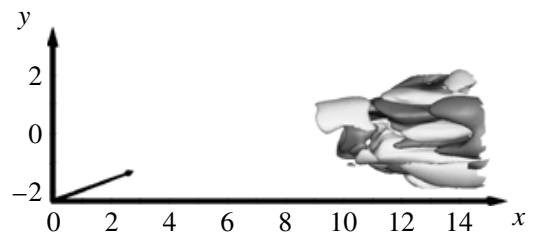

(c)

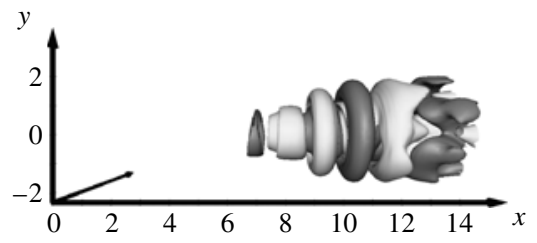

(d)

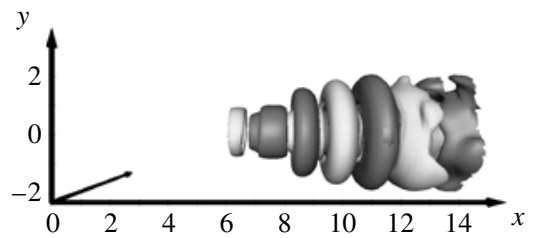

(e)

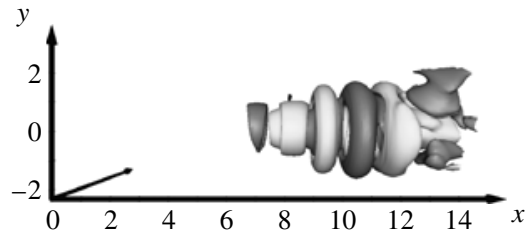

(f)

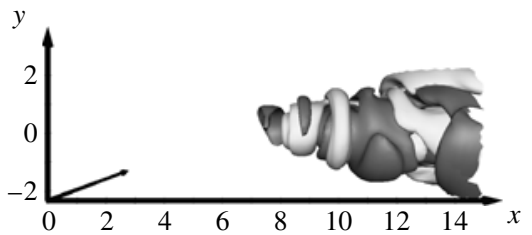

(g)

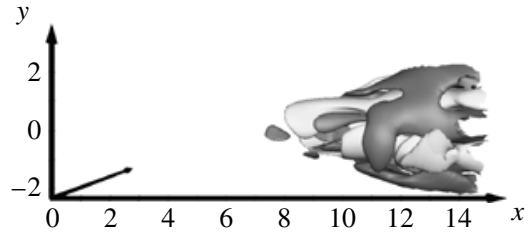

(h)

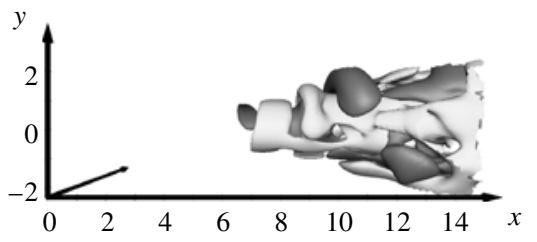

(i)

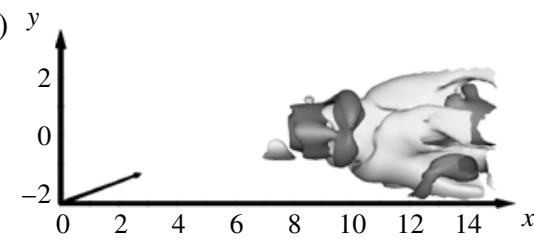

(j)

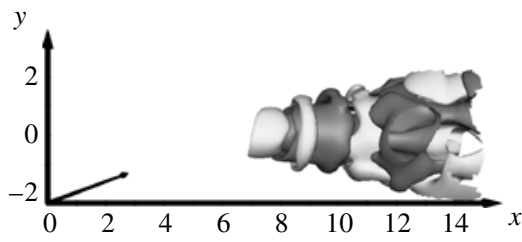

(k)

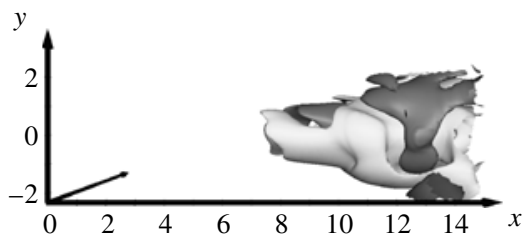

(l)

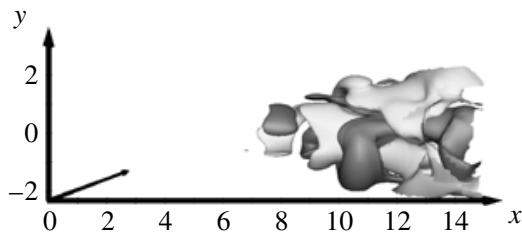

FIgURE 10. $(a-f)$ LR-OID modes and $(g-l)$ LE-OID modes, modes $1-6$ from top to bottom. Displayed are isosurfaces of the streamwise component for positive (light) and negative (dark) values. The grid unit is given by the jet diameter.

is optimized for resolution of turbulent kinetic energy. In the proposed generalization of POD, termed 'observable inferred decomposition' (OID), the resolution of goal functionals is maximized, which are defined by the fluctuation level of linearly related observables. The OID is applied to three configurations to perform goal-oriented dimension reduction: 
(i) In the case of a two-dimensional cylinder wake flow with $R e=100$, the fluctuation levels of the observable lift and drag fluctuation are completely resolved by only one velocity OID mode.

(ii) In a two-dimensional mixing layer with a Reynolds number of 500, four velocity OID modes resolve $85 \%$ of the fluctuation level of an aeroacoustic observable that is monitored by 74 density sensors in the aeroacoustic far field. Thus, a reduction of relevant degrees of freedom is constituted by one order of magnitude as against the typical POD dimension.

(iii) In a three-dimensional $M a=0.9$ jet with a Reynolds number of 3600, 24 velocity OID modes resolves $90 \%$ of the fluctuation level of an aeroacoustic observable that is monitored by 76 pressure sensors in the aeroacoustic far field. Again, a data compression by one order of magnitude is achieved.

For the cylinder wake flow, a subspace of odd and even harmonics is identified by the significant OID mode, respectively, for lift and drag fluctuation. Thus, the wellknown empirical fact that only the odd harmonics correlate with the lift force while only the even harmonics correlate with the drag force fluctuation is thus confirmed by our mathematically rigorous OID approach. For the mixing layer and jet, the most loud flow events due to shear noise are captured by OID. These events qualitatively resemble effects of vortex pairing and amplifying and decaying wavepackets in the case of the mixing layer. In the case of the jet flow, those effects are reminiscent of helical structures, wavy wall mechanisms and vortex rings.

The capability of OID to derive this desired physical understanding fitted for flow control purposes is enabled by a strong coherence of the observable and a dominant, linear coupling of the hydrodynamics with the observable. The OID modes are defined by application of the pseudoinverses of the corresponding linear operator to the POD modes of the observable, such that the efficiency of OID of the hydrodynamic field corresponds to the efficiency of POD of the field of the observable. The well-posedness of this definition is ensured by additional constraints in the form of variational properties, proposing two OID mode variants: for a given resolution of the goal functional, the residual of the flow state attractor and the total kinetic energy is minimized, respectively, in the least-residual OID version (LR-OID) and the least-energetic OID version (LE-OID).

The desired physical understanding benefits reduced-order modelling strategies for control of the aerodynamic and aeroacoustic quantities by systematic flow manipulation. Control goal examples are drag reduction or lift enhancement of wake flows and noise reduction of shear flows. The two OID mode variants are tailored for the purposes of noise control design. A reconstruction of the most probable flow state is supplied by the LR-OID subspace preprocessing efficient observer design. The suppression of the fluctuations of the observable is enabled by strategies pursuing the reduction of the total kinetic energy in the LE-OID subspace, which quantifies the smallest displacement in phase space that a controller has to perform for reduction of the goal functional. Thus, the application of LE-OID to effective control for shear flow noise suppression is encouraged by one of the major OID results of the mixing-layer configuration. Here, only $0.2 \%$ total kinetic energy, identified in the LE-OID subspace, contributes to $85 \%$ of aeroacoustic density fluctuations.

Via OID, a unifying framework of low-order empirical Galerkin expansions is provided. For instance, the capability of the extended POD (EPOD) approach is completely absorbed by the LR-OID variant and upgraded by the additional OID variant of LE-OID, furthermore enabling control design. Moreover, the balanced POD approach (BPOD) enabling the empirical computation of the balanced truncation 
(a)

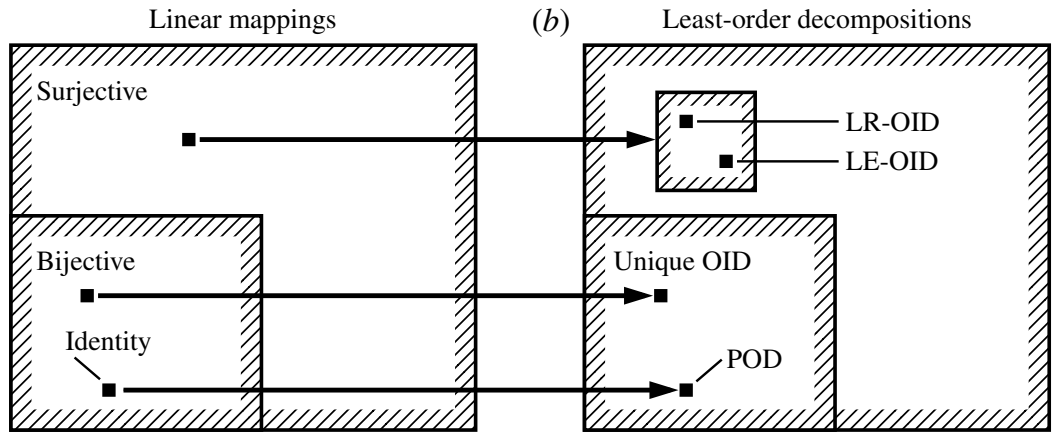

FIGURE 11. Principle of OID design. Any goal-oriented, least-order decomposition (b) is derived from the respective linear mapping (2.12) (a) via the optimally resolved goal functional (2.13). Thus, the basic design parameter is represented by the linear mapping. Surjective mappings exclude any dependence of the observable on quantities other than the (hydrodynamic) attractor. OID is uniquely defined for any linear, bijective mapping. This includes POD as a special case of OID based on the identity map. Additional variational properties can be chosen as a further intrinsic design option for each linear, surjective but not bijective mapping. Here, two OID variants are tailored for purposes of observer design (LR-OID) and control design (LE-OID).

follows a similar goal to the OID method: to identify structures most related to observer and control design. The potential advantage of BPOD relies on the additional premise that the flow dynamics can essentially be represented by a stable, linear input-output system. In contrast to BPOD, the OID approach is based solely on kinematic considerations, which can also deal with nonlinearities of the flow dynamics. Like in the OID approach the flow is decomposed effectively via BPOD, enabled by a modification of the inner product and an error-optimal projection for mode construction. Of course, a meaningful linear coupling of hydrodynamics and the observable (output) is assumed in both approaches, in BPOD as well as in OID.

It should be noted that a large class of least-order decompositions is based on the design of a bilinear form serving as an inner product - at least in a suitable attractor subspace. This decomposition class is completely integrated in the OID technique. These bilinear forms are identified by OID products (2.20), defining the optimal property of the decomposition. Here, the OID induces weights in the bilinear form via the standard inner product of the linearly related observables. Alternatively, these weights can be chosen directly (see Rowley, Colonius \& Murray 2004; Rowley 2005) or by design of optimal control functionals (see Tröltzsch 2005). Via the null space of the bilinear form, design flexibility of the 'observable' OID subspace is provided, enabling OID variants like LR- and LE-OID tailored for purposes of flow control.

OID contains a broad design flexibility, as demonstrated in figure 11: the (hydrodynamic) attractor and the observable may be replaced by any physical quantities fulfilling the OID assumptions for definition of the linear mapping (2.12). This makes OID attractive for future applications to a wide variety of physical problems beyond the application range of POD.

In summary, the OID possesses the following advantages compared to POD: (i) design flexibility, owing to the choice of the observable and the variational property; (ii) extraction of goal-related attractor subspaces with dimensions representing only a fraction of the number of modes necessary for POD; (iii) physical intuition of the 
key processes indicated by the resulting OID modes; (iv) preprocessing for efficient observer and control design; and (v) many conditional sampling techniques (see e.g. Hileman et al. 2005) can be formulated with less bias in OID. As the main OID assumption, linear modelling enables the identification of the attractor subspaces most related to the observables, in a similar spirit to the BPOD approach for stable, linear input-output systems.

Part of our current research is focused on modelling of the dynamics in the OID subspaces and the implementation of actuation, targeting strategies for closed-loop control for several shear flow configurations. These considerations are based on POD Galerkin models extracted from experimental and numerical flow data and calibrated to the flow attractor. We are currently pursuing flow control using a reduced-order model based on turbulence closure (see Noack et al. 2008, 2010; Noack \& Niven 2012) and OID for noise control design (see Schlegel et al. 2009).

\section{Acknowledgements}

The authors acknowledge the funding and excellent working conditions of the DFGCNRS Research Group FOR 508 'Noise Generation in Turbulent Flows', and of the Chaire d'Excellence 'Closed-Loop Control of Turbulent Shear Flows Using ReducedOrder Models' (TUCOROM) of the French Agence Nationale de la Recherche (ANR) and hosted by Institut $\mathrm{P}^{\prime}$. We appreciate valuable stimulating discussions with $\mathrm{B}$. Ahlborn, J.-P. Bonnet, J. Borée, L. Brizzi, P. Comte, L. Cordier, J. Delville, H. Eckelmann, D. Eschricht, M. Farge, C. Franzke, W. K. George, H.-C. Hege, M. Meinke, C.-D. Munz, U. Rist, B. Rummler, K. Schneider, J. Sesterhenn, L. M. Schlegel, A. Spohn, O. Stalnov, G. Tadmor, F. Thiele, C. Tinney, M. Wänström and T. Weinkauf, as well as the local TU Berlin team, R. King, M. Luchtenburg, M. Pastoor and J. Scouten. This work was supported by the Deutsche Forschungsgemeinschaft (DFG) under grants NO. 258/1-1, NO. 258/2-3, SCHL 586/1-1 and SCHL 586/2-1. We thank Hermine Freienstein-Witt for generous additional sources. Part of this work was performed during the Second European Forum on Flow Control, which was supported by AIRBUS through the CAFEDA Research Program, and which took place at the Laboratoire d'Études Aérodynamique, Poitiers, from May to July 2006. The three-dimensional flow visualization has been performed with Amira Software (Zuse Institute, Berlin). We are grateful for outstanding computer and software support from A. Morel, M. Franke and L. Oergel.

\section{Appendix. OID mode variants and OID structures}

The purpose of the appendix is twofold. Firstly, OID variants are mathematically rigorously introduced using optimal properties of projections onto OID subspaces. For reasons of simplicity, the OID variants are first introduced for the Euclidean space of the POD coefficients in $\S$ A.1 before they are defined for unfiltered fields of hydrodynamics and observable in $\S$ A.2. Secondly, OID structures resulting from OID analysis are proposed in $\S$ A.3, the kinematic counterparts of observable structures that are defined in control theory as eigenstructures of the observability Gramian.

\section{A.1. OID mode variants in POD representation}

The starting point of this subsection is the non-uniqueness of the pseudoinverse (see $\S 2.3$ ). Hence, the OID modes $\boldsymbol{u}_{i}^{A}$ and the vector-valued $\boldsymbol{a}_{i}^{u}$ are not well defined at first. For a unique definition of the OID modes, OID subspaces representing the linear span of the OID modes (i.e. the subspace of all linear combinations of the OID modes) are specified using optimal properties. In this subsection, the OID method is formulated 


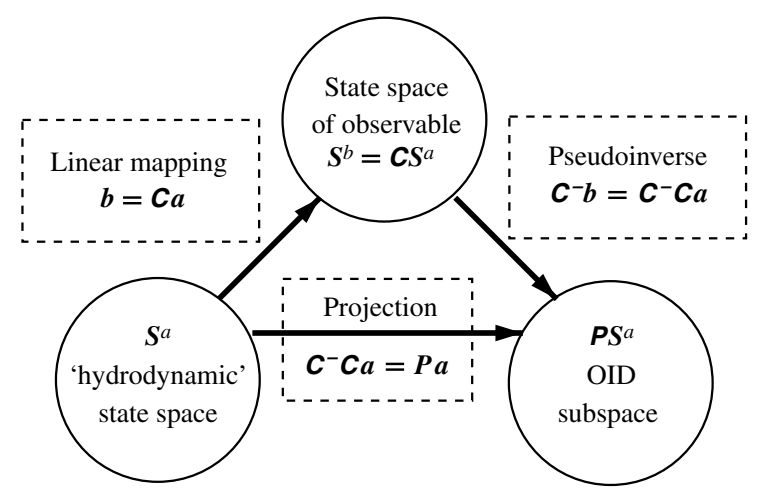

FIGURE 12. Projections onto OID subspaces. The observable is determined from the hydrodynamic data via the linear mapping (2.8) at any time (left arrow). In the case of a dimension defect, only a part of the hydrodynamic quantity is reconstructible by application of a pseudoinverse to the observable (right arrow). This part is specified by the choice of OID subspace: the pseudoinverse is uniquely determined by a particular projection onto this OID subspace (bottom arrow).

in the Euclidean spaces $S^{a}$ and $\boldsymbol{S}^{b}$ of the POD coefficients $\boldsymbol{a}$ and $\boldsymbol{b}$ defined via the representations (2.2) and (2.4).

The OID subspace $\boldsymbol{P S} \boldsymbol{S}^{a}:=\boldsymbol{C}^{-} \boldsymbol{S}^{b}$ represents the subspace of $\boldsymbol{S}^{a}$ reconstructible from the observable using a given pseudoinverse $\boldsymbol{C}^{-}$(see figure 12), which is one-to-one related to the projection $\boldsymbol{P}$,

$$
\boldsymbol{P}:=\boldsymbol{C}^{-} \boldsymbol{C}
$$

The idempotence of $\boldsymbol{P}$ (i.e. $\boldsymbol{P}^{2}=\boldsymbol{P}$ ) is directly proven by the definition of the pseudoinverse $\boldsymbol{C}^{-}$.

Once the projection $\boldsymbol{P}$ is chosen, the pseudoinverse, the OID subspace and the OID modes are uniquely determined. The most important property of $\boldsymbol{P}$ is constituted by the conservation of the observable via the linear mapping (2.8) applied to the projected parts of the hydrodynamic quantity

$$
C P a=C C^{-} C a=C a=b .
$$

The projection $\boldsymbol{P}$ is not necessarily orthogonal, i.e. usually the angle between the projection direction and the OID subspace is oblique (see Example A.1 and figure 13).

In the following, OID subspaces of the hydrodynamic state space are distilled by projections, each defining a respective OID variant. Two projections are selected. While the residual of the projected part of the hydrodynamic ensemble is minimized by the first projection, the vector length of the projection representing the 'total kinetic energy' is minimized by the second projection.

The residual of the hydrodynamic quantity is minimized by the 'least-residual projection' $\boldsymbol{P}^{Z}$, the argument of the minimization problem

$$
\min _{\boldsymbol{P}: \boldsymbol{P}=\boldsymbol{C}^{-} \boldsymbol{c}}\left\langle\|\boldsymbol{a}(t)-\boldsymbol{P a}(t)\|^{2}\right\rangle
$$

where the Euclidean vector norm is denoted by $\|\cdot\|$. 'Least-residual' OID modes (LROID modes) are defined by definition (2.15) using the pseudoinverse given by (A 1) with $\boldsymbol{P}=\boldsymbol{P}^{Z}$. 

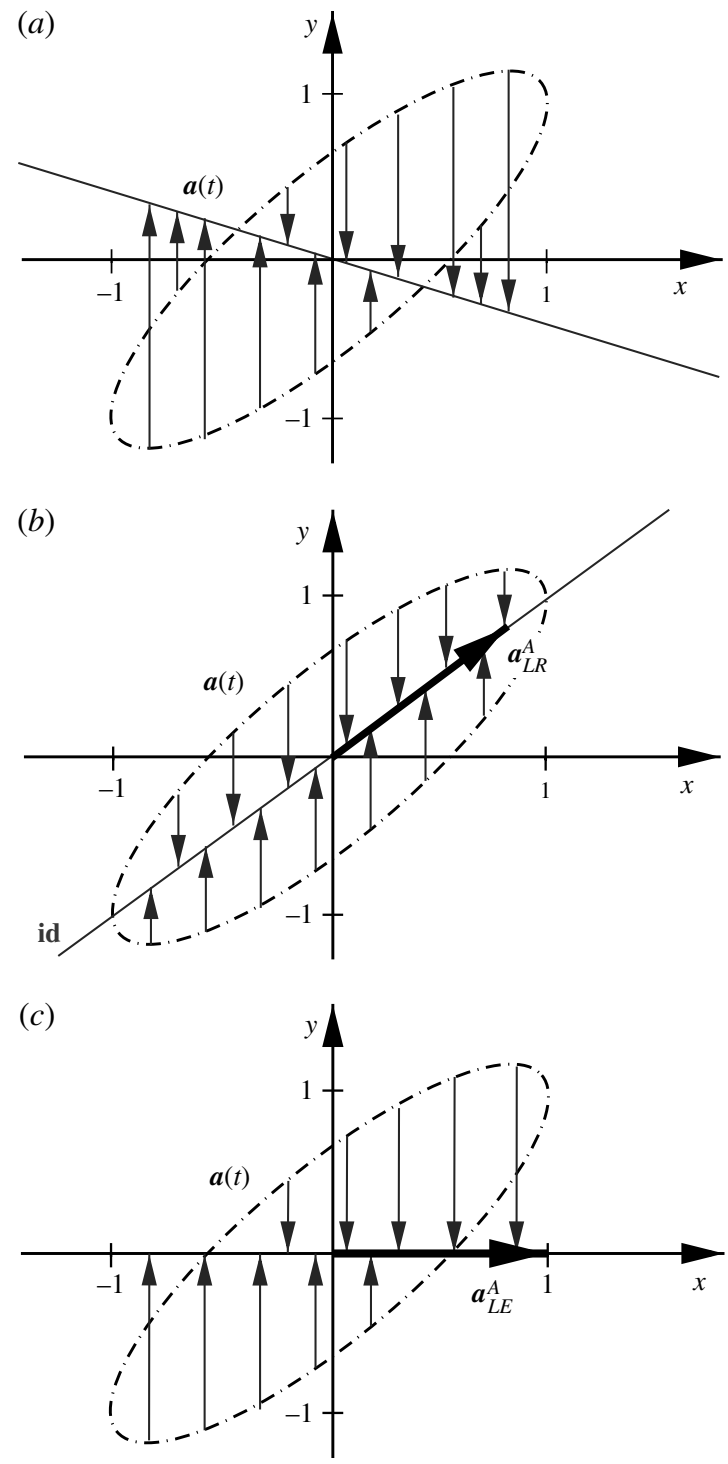

FIgURE 13. Principle of Example A.1. The ensemble of the hydrodynamic data is represented by the ellipse (dashed-dotted line). The observable is represented by the $x$ coordinate of this ellipse. (a) By any projection of the form $\boldsymbol{P}=\boldsymbol{C}^{-} \boldsymbol{C}$, the ellipse is projected onto an OID subspace in the vertical direction, thus conserving the observable. $(b)$ Under the latter side constraint, the linear least-squares fit is determined from the projection onto the OID subspace of LR-OID, which is given by the line of identity. (c) Similarly, the Euclidean vector norm is minimized by the orthogonal projection onto the abscissa representing the OID subspace of LE-OID.

The norm of the projection is minimized by the 'least-energetic projection' $\boldsymbol{P}^{C}$, the argument of the minimization problem

$$
\min _{\boldsymbol{P}: \boldsymbol{P}=\boldsymbol{C}^{-} \boldsymbol{c}}\left\langle\|\boldsymbol{P a}(t)\|^{2}\right\rangle
$$


'Least-energetic' OID modes (LE-OID modes) are obtained again from definition (2.15) and (A 1) employing the projection $\boldsymbol{P}=\boldsymbol{P}^{C}$.

EXAMPLE A.1. Let the hydrodynamic flow data and the (one-dimensional) observable be given by

$$
\boldsymbol{a}=\left[\begin{array}{l}
x(t) \\
y(t)
\end{array}\right]=\left[\begin{array}{c}
\sin (2 \pi t) \\
\sin (2 \pi t)+\cos (2 \pi t)
\end{array}\right], \quad b=\sin (2 \pi t),
$$

for all $t \in \mathbb{R}$. Identification of the linear mapping (2.8) determines the linear mapping $\boldsymbol{C}=[1,0]$. Any projection fulfilling the constraint $\boldsymbol{P}=\boldsymbol{C}^{-} \boldsymbol{C}$ is educible by

$$
\boldsymbol{P}=\left[\begin{array}{ll}
1 & 0 \\
\beta & 0
\end{array}\right]
$$

with arbitrary $\beta \in \mathbb{R}$. Hence, the corresponding pseudoinverse and OID subspace are given by

$$
\boldsymbol{C}^{-}=\left[\begin{array}{l}
1 \\
\beta
\end{array}\right] \quad \text { and } \quad[x, y] \boldsymbol{C}^{-}=0,
$$

respectively. Thus, all straight lines crossing the origin except the ordinate represent candidates for the selection of an OID subspace (see figure 13).

The least-residual projection $\boldsymbol{P}^{Z}$ is computed from minimum problem (A 3). Using (A 6) it is transformed to the minimum problem

$$
\min _{\beta \in \mathbb{R}}(1-\beta)^{2},
$$

which is solved at $\beta=1$. Thus,

$$
\boldsymbol{P}^{Z}=\left[\begin{array}{ll}
1 & 0 \\
1 & 0
\end{array}\right] \quad \text { and } \quad \boldsymbol{C}^{-}=\left[\begin{array}{l}
1 \\
1
\end{array}\right]
$$

The OID subspace is represented by the line of identity (see figure 13). Hence $Q^{A}(\boldsymbol{a})$ is completely resolved by one LR-OID mode, given, after normalization, by the vector

$$
\boldsymbol{a}_{L R}^{A}=\frac{1}{\sqrt{2}}\left[\begin{array}{l}
1 \\
1
\end{array}\right] \text {. }
$$

Similarly,

$$
\min _{\beta \in \mathbb{R}}\left(\frac{1}{2}+\beta^{2}\right)
$$

is derived from the minimum problem (A4). The minimum is reached at $\beta=0$. Thus, the least-energetic projection operator and its corresponding pseudoinverse are obtained as

$$
\boldsymbol{P}^{C}=\left[\begin{array}{ll}
1 & 0 \\
0 & 0
\end{array}\right] \quad \text { and } \quad \boldsymbol{C}^{-}=\left[\begin{array}{l}
1 \\
0
\end{array}\right]
$$




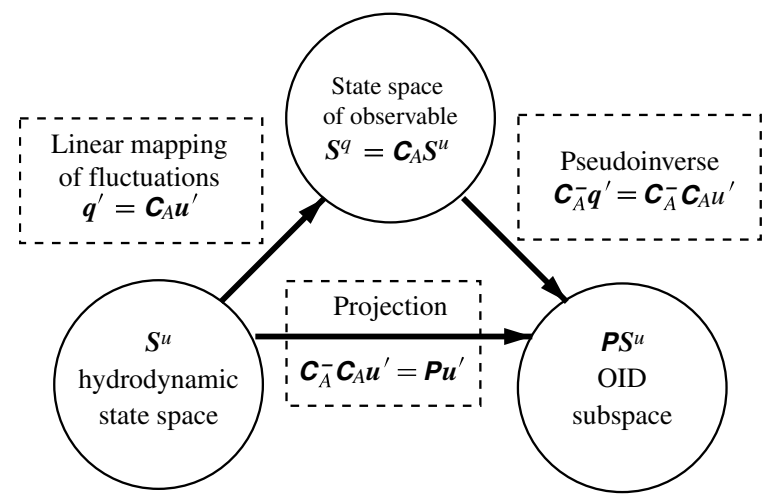

FIGURE 14. Projections onto OID subspaces as an OID principle. Same as figure 12, but based on the generalized formulation for fields.

The OID subspace is represented by the abscissa in figure 13. Thus, the corresponding LE-OID mode is given by the vector

$$
\boldsymbol{a}_{L E}^{A}=\left[\begin{array}{l}
1 \\
0
\end{array}\right] .
$$

\section{A.2. OID mode variants}

For a unique definition of the OID modes $\boldsymbol{u}^{A}$, the concept of the OID subspace of the previous subsection is generalized to subspaces of the hydrodynamic attractor, represented again by the linear span of the OID modes. The OID subspace $\boldsymbol{P} \boldsymbol{S}^{u}:=\boldsymbol{C}_{A}^{-} \boldsymbol{S}^{q}$ represents the subspace of $\boldsymbol{S}^{u}$ reconstructible from the observable using a given pseudoinverse $\boldsymbol{C}_{A}^{-}$(see figure 14), which is one-to-one related to a projection operator $\boldsymbol{P}$ similar to that in (A 1) via

$$
\boldsymbol{P}=\boldsymbol{C}_{A}^{-} \boldsymbol{C}_{A}
$$

Analogously to the arguments of (A2), the conservation of the fluctuations of the observable under application of any projection of the form (A 9) is shown.

'Observable' OID subspaces of the hydrodynamic state space are distilled by one of the two projections from the previous subsection obeying the following two variational properties. Two OID mode variants are defined by the latter, tailored for purposes of observer and control design. As in the previous subsection, these variants are termed 'LE-OID' and 'LR-OID' in the following.

The flow attractor residual is minimized by the 'least-residual projection' $\boldsymbol{P}^{Z}$, and $\boldsymbol{P}^{Z}$ is defined as in the minimization problem (A 3) but using the norm $\|\cdot\|_{\Omega}$ induced by the inner product $(\cdot, \cdot)_{\Omega}$ instead of the Euclidean vector norm $\|\cdot\|$. The reconstruction of the most probable flow state from a given observable is enabled by the 'leastresidual' OID modes (LR-OID modes), given from (2.14) using the pseudoinverse, which is uniquely defined by (A 9) with projection $\boldsymbol{P}^{Z}$. Thus, LR-OID modes provide a basis for observer design.

The level of the projected hydrodynamic fluctuations is minimized by the 'leastenergetic projection' $\boldsymbol{P}^{C}$, and $\boldsymbol{P}^{C}$ is defined by the minimization problem (A 4), using 


$$
\text { OID subspace }
$$

LR-OID

$$
\begin{gathered}
\text { correlated structures } \\
\boldsymbol{u}^{\mathscr{O}}=\boldsymbol{P}^{Z} \boldsymbol{u}^{\prime}: \forall \boldsymbol{x} \in \Omega, \boldsymbol{y} \in \Gamma ; \\
\left\langle\boldsymbol{u}^{\mathscr{O}}(\boldsymbol{x}, t), \boldsymbol{q}^{\prime}(\boldsymbol{y}, t+\tau)\right\rangle \\
=\left\langle\boldsymbol{u}^{\prime}(\boldsymbol{x}, t), \boldsymbol{q}^{\prime}(\boldsymbol{y}, t+\tau)\right\rangle
\end{gathered}
$$

LE-OID

$$
\begin{aligned}
& \text { generating structures } \\
& \begin{aligned}
\boldsymbol{u}^{\mathscr{O}} & =\boldsymbol{P}^{C} \boldsymbol{u}^{\prime} \forall t ; \boldsymbol{C}_{A} \boldsymbol{u}^{\mathscr{O}}(t) \\
& =\boldsymbol{C}_{A} \boldsymbol{u}^{\prime}(t)=\boldsymbol{q}^{\prime}(t+\tau)
\end{aligned}
\end{aligned}
$$

OID residuum

uncorrelated structures

$$
\begin{gathered}
\boldsymbol{u}^{\mathscr{N}}: \forall \boldsymbol{x} \in \Omega, \boldsymbol{y} \in \Gamma ; \\
\left\langle\boldsymbol{u}^{\mathscr{N}}(\boldsymbol{x}, t), \boldsymbol{q}^{\prime}(\boldsymbol{y}, t+\tau)\right\rangle=0
\end{gathered}
$$

non-generating structures

$\boldsymbol{u}^{\mathscr{N}}: \forall t ; \boldsymbol{C}_{A} \boldsymbol{u}^{\mathscr{N}}(t)=\mathbf{0}$

TABLE 2. Properties of OID structures and their residuals in LR-OID and LE-OID. In LR-OID, only the OID structures contribute to the correlation of hydrodynamic fluctuations and the fluctuations of the observable (correlated structures), while the OID residuals are uncorrelated to the fluctuations of the observable (non-correlated structures). In LEOID, only the OID structures contribute to the linear mapping (2.12) from hydrodynamic fluctuations to fluctuations of the observable (generating structures), while the OID residuals are situated in the null space of the linear mapping (non-generating structures).

again the norm $\|\cdot\|_{\Omega}$ instead of $\|\cdot\|$. 'Least-energetic' OID modes (LE-OID modes) are obtained from the least-energetic projection $\boldsymbol{P}^{C}$.

\section{A.3. Filtering OID structures}

POD is well known to act as a filter to separate coherent structures, represented by the POD approximation (2.2), from their residuum of stochastic structures. Analogously in OID, hydrodynamic fluctuations are decomposed into OID structures and their residual. As an illustration, OID for an aeroacoustic observable distils 'noisy' and 'silent' flow structures and filtered counterparts 'loud' and 'quiet' flow structures to provide a physical understanding for noise control.

First of all, the OID subspace and its orthogonal complement decompose the hydrodynamic fluctuations orthogonally into an OID part (the 'noisy' part) and its residual (the 'silent' part) $\boldsymbol{u}^{\prime}=\boldsymbol{u}^{\mathscr{O}}+\boldsymbol{u}^{\mathscr{N}}$, where $\boldsymbol{u}^{\mathscr{O}}(t)=\boldsymbol{P} \boldsymbol{u}^{\prime}(t)$ represents the OID structures, and $\boldsymbol{u}^{\mathscr{N}}(t)=(\boldsymbol{I}-\boldsymbol{P}) \boldsymbol{u}^{\prime}(t)$ the OID residual. The physical meanings of this decomposition are outlined in table 2 for both OID variants.

Commonly, only a small subset of modes is utilized in POD, e.g. the smallest subset needed to resolve $90 \%$ total kinetic energy (see Holmes et al. 1998). Analogously, we consider only a subset of the set of OID modes $\left\{\boldsymbol{u}_{i}^{A}\right\}_{i=1}^{M}$, e.g. the smallest subset to resolve $90 \%$ of the correlated goal functional. Thus, we define a filtered counterpart of the OID structures (the 'loud' part) by

$$
\boldsymbol{u}^{\mathscr{M}}(\boldsymbol{x}, t)=\sum_{i=1}^{L} a_{i}^{A}(t) \boldsymbol{u}_{i}^{A}(\boldsymbol{x}, t),
$$

with $L \leqslant M$, and a filtered counterpart of the OID residual (the 'quiet' part) by

$$
\boldsymbol{u}^{\mathscr{H}}(\boldsymbol{x}, t)=\boldsymbol{u}^{\prime}(\boldsymbol{x}, t)-\boldsymbol{u}^{\mathscr{M}}(\boldsymbol{x}, t) .
$$

The properties of OID structures and OID residual shown in table 2 can be transferred to the filtered equivalents. 


\section{REFERENCES}

Afanasiev, K. 2003 Stabilitätsanalyse, niedrigdimensionale Modellierung und optimale Kontrolle der Kreiszylinderumströmung [Stability analysis, low-dimensional modelling, and optimal control of the flow around a circular cylinder]. PhD Thesis, Technische Universität Dresden, Germany.

Ben-Israel, A. \& Greville, T. N. E. 2003 Generalized Inverses: Theory and Applications, vol. 15, 2nd edn. CMS Books in Mathematics, Springer.

Bergmann, M., Cordier, L. \& Brancher, J.-P. 2005 Optimal rotary control of the cylinder wake using proper orthogonal decomposition reduced order model. Phys. Fluids 17, 1-21.

BorÉE, J. 2003 Extended proper orthogonal decomposition: a tool to analyse correlated events in turbulent flows. Exp. Fluids 35, 188-192.

Cavalieri, A. V. G., Daviller, G., Comte, P., Jordan, P., Tadmor, G \& Gervais, Y. $2011 a$ Using large eddy simulation to explore sound source mechanisms in jets. J. Sound Vib. 330, 4098-4113.

Cavalieri, A. V. G., Jordan, P., Agarwal, A. \& Gervais, Y. $2011 b$ Jittering wavepacket models for subsonic jet noise. J. Sound Vib. 330, 4474-4492.

Cavalieri, A. V. G., Jordan, P., Gervais, Y. \& Colonius, T. 2011c Axisymmetric superdirectivity in subsonic jets. In 17th AIAA/CEAS Aeroacoustics Conference. AIAA Paper 2011-2743.

Cazemier, W., Verstappen, R. W. C. P. \& Veldman, A. E. P. 1998 Proper orthogonal decomposition and low-dimensional models for driven cavity flows. Phys. Fluids 7, $1685-1699$.

Coiffet, F., Jordan, P., Delville, J., Gervais, Y. \& Ricaud, F. 2006 Coherent structures in subsonic jets: a quasi-irrotational source mechanism? Intl J. Aeroacoust. 5 (1), 67-89.

Crighton, D. G. 1975 Basic principles of aerodynamic noise generation. Prog. Aerosp. Sci. 16, 31-96.

Crighton, D. G. \& Huerre, P. 1990 Shear layer pressure fluctuations and superdirective acoustic sources. J. Fluid Mech. 220, 355-368.

Ffowcs Williams, J. E. 1963 The noise from turbulence convected at high speed. Phil. Trans. R. Soc. Lond. A 231, 505-514.

FRANZKE, C. \& MAJDA, A. J 2006 Low order stochastic mode reduction for a prototype atmospheric GCM. J. Atmos. Sci. 63 (2), 457-479.

FreUnd, J. 2001 Noise sources in a low Reynolds number turbulent jet at Mach 0.9. J. Fluid Mech. 438, 277-305.

Freund, J. \& COLOniUs, T. 2002 POD analysis of sound generation by a turbulent jet. AIAA Paper 2002-0072.

Freund, J. \& Colonius, T. 2009 Turbulence and sound-field POD analysis of a turbulent jet. Intl J. Aeroacoust. 8 (4), 337-354.

Gerhard, J., Pastoor, M., King, R., Noack, B. R., Dillmann, A., Morzyński, M. \& TADMOR, G. 2003 Model-based control of vortex shedding using low-dimensional Galerkin models, AIAA Paper 2003-4262.

Gröschel, E., Schröder, W., Schlegel, M., Scouten, J., NoACK, B. R. \& Comte, P. 2007 Reduced-order analysis of turbulent jet flow and its noise source. ESAIM: Proc. 16, 33-50.

Gröschel, E., Schröder, W., Renze, P., Meinke, M. \& Comte, P. 2008 Noise prediction for a turbulent jet using different hybrid methods. Comput. Fluids 37, 414-426.

Guj, G., Carley, C. \& Camussi, R. 2003 Acoustic identification of coherent structures in a turbulent jet. J. Sound Vib. 259 (5), 1037-1065.

Hileman, J. I., Caraballo, E. J., Thurow, B. S. \& Samimy, M. 2004 Differences in dynamics of an ideally expanded Mach 1.3 jet during noise generation and relative quiet periods. AIAA Paper 2004-3015.

Hileman, J. I., Thurow, B. S., Caraballo, E. J. \& Samimy, M. 2005 Large-scale structure evolution and sound emission in high-speed jets: real-time visualization with simultaneous acoustic measurements. J. Fluid Mech. 544, 277-307. 
Hoarau, C., Borée, J., Laumonier, J. \& Gervais, Y. 2006 Analysis of the wall pressure trace downstream of a separated region using extended proper orthogonal decomposition. Phys. Fluids 18, 055107.

Holmes, P., Lumley, J. L. \& Berkooz, G. 1998 Turbulence, Coherent Structures, Dynamical Systems and Symmetry. Cambridge University Press.

Howe, M. S. 2003 Theory of Vortex Sound. Cambridge University Press.

Jordan, P. \& Gervais, Y. 2008 Subsonic jet aeroacoustics: associating experiment, modelling and simulation. Exp. Fluids 44, 1-21.

Jordan, P., Schlegel, M., Stalnov, O., Noack, B. R. \& Tinney, C. E. 2007 Identifying noisy and quiet modes in a jet. In 13th AIAA/CEAS Aeroacoustics Conference. AIAA Paper 2007-3602.

Jørgensen, B. H., Sørensen, J. N. \& Brøns, M. 2003 Low-dimensional modelling of a driven cavity flow with two free parameters. Theor. Comput. Fluid Dyn. 16, 299-317.

Juvé, D., Sunyach, M. \& Comte-Bellot, G. 1980 Intermittency of the noise emission in subsonic cold jets. J. Sound Vib. 71 (3), 319-332.

Lall, S., Marsden, J. E. \& GlavašKi, S. 1999 Empirical model reduction of controlled nonlinear systems. In Proceedings of the 14th IFAC World Congress, vol. F, pp. 473-478. International Federation of Automatic Control (IFAC), Laxenburg, Austria.

LAll, S., MARsden, J. E. \& GlavašKi, S. 2002 A subspace iteration approach to balanced truncation for model reduction of nonlinear control systems. Intl J. Robust Nonlinear Control 12, 519-535.

Laurendeau, E., Jordan, P., Bonnet, J. P., Delville, J., Parnaudeau, P. \& Lamballais, E. 2008 Subsonic jet noise reduction by fluidic control: the interaction region and the global effect. Phys. Fluids 20, 101519.

LeE, H. K. \& RibneR, H. S. 1972 Direct correlation of noise and flow of a jet. J. Acoust. Soc. Am. $52(5), 1280-1290$.

Lighthill, M. J. 1952 On sound generated aerodynamically: I. General theory. Proc. R. Soc. Lond. A 211, 564-587.

Luchtenburg, D. M., Günther, B., Noack, B. R., King, R. \& Tadmor, G. 2009 A generalized mean-field model of the natural and high-frequency actuated flow around a high-lift configuration. J. Fluid Mech. 623, 283-316.

Lugt, H. J. 1996 Introduction to Vortex Theory. Vortex Flow Press.

Maurel, S., Borée, J. \& Lumley, J. L. 2001 Extended proper orthogonal decomposition: application to jet/vortex interaction. Flow Turbul. Combust. 67, 125-136.

Meinke, M., Schröder, W., Krause, E. \& Rister, T. R. 2002 A comparison of second- and sixth-order methods for large-eddy simulations. Comput. Fluids 21, 695-718.

MoRZYŃSKI, M. 1987 Numerical solution of Navier-Stokes equations by the finite element method. In Proceedings of SYMKOM 87, Compressor and Turbine Stage Flow Path - Theory and Experiment, Reports of the Institute of Turbomachinery 527, Cieplne Maszyny Przeptwowe 94, pp. 119-128. Technical University of Łódź.

Morzyński, M., Stankiewicz, W., Noack, B. R., King, R., Thiele, F. \& Tadmor, G. 2007 Continuous mode interpolation for control-oriented models of fluid flow. In Active Flow Control: Papers Contributed to the Conference 'Active Flow Control 2006', Berlin, Germany, September 27 to 29, 2006 (ed. R. King), Notes on Numerical Fluid Mechanics and Multidisciplinary Design, vol. 95, pp. 260-278. Springer.

Noack, B. R., Afanasiev, K., Morzyński, M., Tadmor, G. \& Thiele, F. 2003 A hierarchy of low-dimensional models for the transient and post-transient cylinder wake. J. Fluid Mech. 497, 335-363.

NoAck, B. R., Morzyński, M. \& TAdmor, G. (Eds) 2011 Reduced-Order Modelling for Flow Control. CISM Courses and Lectures, vol. 528. Springer.

NoAck, B. R., Papas, P. \& Monkewitz, P. A. 2005 The need for a pressure-term representation in empirical Galerkin models of incompressible shear flows. J. Fluid Mech. 523, 339-365.

Nohck, B. R., Schlegel, M., Ahlborn, B., Mutschke, G., Morzyński, M., Comte, P. \& TADMOR, G. 2008 A finite-time thermodynamics of unsteady fluid flows. J. Non-Equilib. Thermodyn. 33 (2), 103-148. 
NoAck, B. R., Schlegel, M., Morzyński, M. \& TAdmor, G. 2010 System reduction strategy for Galerkin models of fluid flows. Intl J. Numer. Meth. Fluids 63 (2), 231-248.

NoAck, B. R. \& Niven, R. K. 2012 Maximum entropy closure for a Galerkin model of an incompressible periodic wake. J. Fluid Mech. (in press).

Panda, J., Seasholtz, R. G. \& Elam, K. A. 2005 Investigation of noise sources in high-speed jets via correlation measurements. J. Fluid Mech. 537, 349-385.

Pastoor, M., Henning, L., Noack, B. R., King, R. \& Tadmor, G. 2008 Feedback shear layer control for bluff body drag reduction. J. Fluid Mech. 608, 161-196.

PiCARD, C. \& Delville, J. 2000 Pressure velocity coupling in a subsonic round jet. Intl J. Heat Fluid Flow 21, 359-364.

Protas, B. \& Wesfreid, J. E. 2003 On the relation between the global modes and the spectra of drag and lift in periodic wake flows. C. R. Méc. 331, 49-54.

REMPfER, D. \& FASEL, H. F. 1994 Dynamics of three-dimensional coherent structures in a flat-plate boundary layer. J. Fluid Mech. 275, 257-283.

Rodriguez Alvarez, D., Samanta, A., Cavalieri, A. V. G., Colonius, T. \& Jordan, P. 2011 Parabolized stability equation models for predicting large-scale mixing noise of turbulent round jets. In 17th AIAA/CEAS Aeroacoustics Conference. AIAA Paper 2011-2743.

Rowley, C. W. 2005 Model reduction for fluids using balanced proper orthogonal decomposition. Intl J. Bifurcation Chaos 15 (3), 997-1013.

Rowley, C. W., Colonius, T. \& Murray, R. M. 2004 Model reduction for compressible flows using POD and Galerkin projection. Physica D 189 (1-2), 115-129.

Samimy, M., Kim, J.-H., Kastner, J., Adamovich, I. \& UtKin, Y. 2007 Active control of a Mach 0.9 jet for noise mitigation using plasma actuators. AIAA J. 45 (4), 890-901.

SCHAFFAR, M. 1979 Direct measurements of the correlation between axial in-jet velocity fluctuations and far field noise near the axis of a cold jet. J. Sound Vib. 64 (1), 73-83.

SCHAFFAR, M. \& HANCY, J. P 1982 Investigation of the noise emitting zones of a cold jet via causality correlations. J. Sound Vib. 81 (3), 377-391.

Scharton, T. D. \& White, P. H. 1972 Simple pressure source model of jet noise. J. Acoust. Soc. Am. 52 (1), 399-412.

Schlegel, M., Noack, B. R., Comte, P., Kolomenskiy, D., Schneider, K., Farge, M., Scouten, J., Luchtenburg, D. M. \& TAdMor, G. 2009 Reduced-order modelling of turbulent jets for noise control. In Numerical Simulation of Turbulent Flows and Noise Generation (ed. C. Brun, D. Juvé, M. Manhart \& C.-D. Munz). Notes on Numerical Fluid Mechanics and Multidisciplinary Design, vol. 104, pp. 3-27. Springer.

SEINER, J. M. 1974 The distribution of jet source strength intensity by means of a direct correlation technique. PhD Thesis, Pennsylvania State University, University Park, PA.

SeIner, J. M. \& ReEtoff, G. 1974 On the distribution of source coherency in subsonic jets. AIAA Paper 1974-4.

Siegel, S. G., Seidel, J., Fagley, C., Luchtenburg, D. M., Cohen, K. \& Mclaughlin, T. 2008 Low-dimensional modelling of a transient cylinder wake using double proper orthogonal decomposition. J. Fluid Mech. 610, 1-42.

Sirovich, L. 1987 Turbulence and the dynamics of coherent structures, Part I: Coherent structures. Q. Appl. Math. XLV, 561-571.

TAM, C. 1998 Jet noise: since 1952. Theor. Comput. Fluid Dyn. 10, 393-405.

Townsend, A. A. 1956 The Structure of Turbulent Shear Flow. Cambridge University Press.

TRÖLTZsCH, F. 2005 Optimale Steuerung partieller Differentialgleichungen: Theorie, Verfahren und Anwendungen. Vieweg.

WEI, M. 2004 Jet noise control by adjoint-based optimization. PhD Thesis, University of Illinois at Urbana-Champaign, IL.

Wei, M. \& Freund, J. 2006 A noise-controlled free shear flow. J. Fluid Mech. 546, 123-152.

WeI, M. \& Rowley, C. W. 2009 Low-dimensional models of a temporally evolving free shear layer. J. Fluid Mech. 618, 113-134.

WILlCOX, K. 2006 Flow sensing and estimation via the gappy proper orthogonal decomposition. Comput. Fluids 35 (2), 208-226. 
Willcox, K. \& Megretski, A. 2005 Fourier series for accurate, stable, reduced-order models in large-scale applications. SIAM J. Sci. Comput. 26 (3), 944-962.

Willcox, K. \& Peraire, J. 2002 Balanced model reduction via the proper orthogonal decomposition. AIAA J. 40 (11), 2323-2330.

Wu, J.-Z., MA, H.-Y. \& Zhou, M.-D. 2006 Vorticity and Vortex Dynamics. Springer. 